\title{
Cognitive Radio: An Information-Theoretic Perspective
}

\author{
Aleksandar Jovičić and Pramod Viswanath *†
}

May 11, 2009

\begin{abstract}
We consider a communication scenario in which the primary and the cognitive radios wish to communicate to different receivers, subject to mutual interference. In the model that we use, the cognitive radio has non-causal knowledge of the primary radio's codeword. We characterize the largest rate at which the cognitive radio can reliably communicate under the constraint that (i) no rate degradation is created for the primary user, and (ii) the primary receiver uses a single-user decoder just as it would in the absence of the cognitive radio. The result holds in a "low interference" regime in which the cognitive radio is closer to its receiver than to the primary receiver. In this regime, our results are subsumed by the results derived in a concurrent and independent work [24]. We also demonstrate that, in a "high interference" regime, multi-user decoding at the primary receiver is optimal from the standpoint of maximal jointly achievable rates for the primary and cognitive users.
\end{abstract}

Index Terms - Cognitive radio, Costa precoding, dirty-paper coding, interference channel, spectral reuse, wireless networks.

\section{Introduction}

Observing a severe under-utilization of the licensed spectrum, the FCC has recently recommended $[7,8]$ that significantly greater spectral efficiency could be realized by de-

*A. Jovičić is with Qualcomm Flarion Technologies, Bridgewater, NJ. P. Viswanath is with the department of Electrical and Computer Engineering at the University of Illinois at Urbana-Champaign. Email: ajovicic@qualcomm.com, and pramodv@uiuc.edu

${ }^{\dagger}$ This research was supported in part by the National Science Foundation under grant CCR-0312413 and a grant from Motorola Inc. as part of the Motorola Center for Communication. 
ploying wireless devices that can coexist with the incumbent licensed (primary) users, generating minimal interference while somehow taking advantage of the available resources. Such devices could, for instance, form real-time secondary markets [16] for the licensed spectrum holders of a cellular network or even, potentially, allow a complete secondary system to simultaneously operate in the same frequency band as the primary. The characteristic feature of these cognitive radios would be their ability to recognize their communication environment and adapt the parameters of their communication scheme to maximize the quality of service for the secondary users while minimizing the interference to the primary users.

In this paper, we study the fundamental limits of performance of wireless networks endowed with cognitive radios. In particular, in order to understand the ultimate systemwide benefits of the cognitive nature of such devices, we assume that the cognitive radio has non-causal knowledge of the codeword of the primary user in its vicinity ${ }^{1}$; in this, we are motivated by the model proposed in [6]. We address the following fundamental question:

What is the largest rate that the cognitive radio can achieve under the constraint that

(i) it creates no rate degradation for the primary user in its vicinity, and

(ii) the primary receiver uses a single-user decoder, just as it would in the absence of the cognitive radio?

We will refer to these two imperative constraints as the coexistence conditions that a cognitive secondary system must satisfy.

Of central interest to us is the communication scenario illustrated in Fig. 1: The primary user wishes to communicate to the primary base-station $B_{p}$. In its vicinity is a secondary user equipped with a cognitive radio that wishes to transmit to the secondary base-station $B_{s}$. We assume that the cognitive radio has obtained the message of the primary user. The received signal-to-noise ratio of the cognitive radio's transmission at the secondary base-station is denoted by SNR. The transmission of the cognitive radio is also received at $B_{p}$, and the signal-to-noise ratio of this interfering signal is denoted by INR (interference-to-noise ratio). If the cognitive user is close to $B_{p}$, INR could potentially be large.

We identify the largest rate at which the cognitive radio can reliably communicate with its receiver $B_{s}$ under the coexistence conditions and in the "low-interference-gain" regime in which INR $\leq$ SNR. This regime is of practical interest since it models the realistic scenario in which the cognitive radio is closer to $B_{s}$ than to $B_{p}$. Moreover, we

\footnotetext{
${ }^{1}$ Note that this does not imply that the cognitive user can decode the information that the primary user is communicating since there are secure encryption protocols running at the application layer. The decoded codeword is a meaningless stream of bits for the cognitive user.
} 


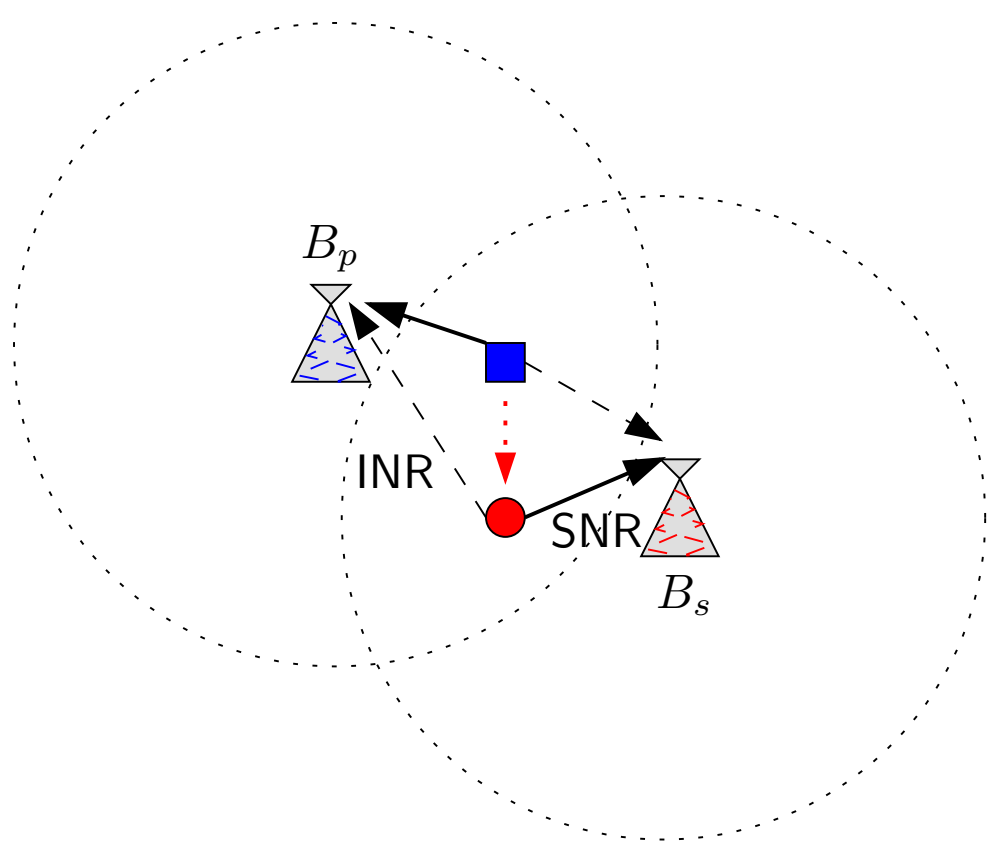

Figure 1: A possible arrangement of the primary and secondary receivers, base-stations $B_{p}$ and $B_{s}$, respectively. The cognitive secondary user is represented by the circle and the primary user is represented by the square. The side-information path is depicted by the dotted line.

show that the capacity achieving strategy is for the cognitive radio to perform precoding for the primary users's codeword and transmit over the same time-frequency slot as that used by the primary radio.

To prove this result, we allow the primary and secondary systems to cooperate and jointly design their encoder-decoder pairs and then show that the optimal communication scheme for this cooperative situation has the property that the primary decoder does not depend on the encoder and decoder used by the secondary system. Under such a joint encoder/decoder design, the cognitive radio channel can be thought of as the classical interference channel [1], [18], [4] but with degraded message sets ${ }^{2}$ : Achievable schemes for this channel have been first studied in [6]. The capacity region of this channel, in the low interference gain regime, has recently been found by an independent and concurrent work [23], [24]. In this paper, we provide an alternative proof for a portion of the capacity region of the same channel. The capacity region under the assumption that both receivers experience high interference has been reported in [13]. A related problem of communicating a single private message along with a common message to each of the receivers has been studied in [14].

In contrast to our results for the low-interference-gain regime, we exhibit a regime in which joint code design is beneficial with respect to the largest set of simultaneously achievable rates of the two radios. We find that, when INR $\gg$ SNR, multi-user decoding

\footnotetext{
${ }^{2}$ The primary radio has only a subset of the messages available to the cognitive radio.
} 
by the primary receiver is required in order to achieve all the rates in the capacity region of the interference channel with degraded message sets.

The rest of this paper is organized as follows. We first introduce the Gaussian cognitive radio channel in Section 2. The capacity of the cognitive radio channel in the lowinterference-gain regime, where INR $\leq$ SNR, is presented in Section 3. The proof is given in Section 3, where we demonstrate a part of the capacity region of the underlying interference channel with degraded message sets which inherently allows for joint code design. This result, along with that of the parallel work by [24], yields the capacity of the Gaussian cognitive radio channel. We then show that the benefit of joint code design becomes apparent in the high-interference-gain regime INR $\gg S N R$; this is done in Section 4.2. Finally, we study the system-level implications of the optimal cognitive communication scheme in Section 5.

\section{The Channel Model and Problem Statement}

\subsection{The cognitive radio channel}

Consider the following communication scenario which we will refer to as the cognitive radio channel.

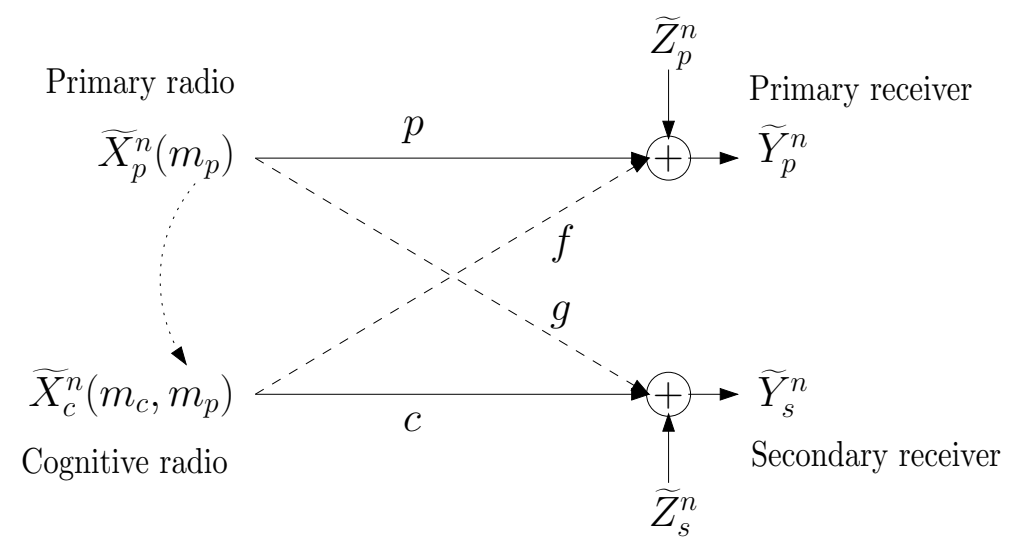

Figure 2: The (Gaussian) cognitive radio channel after $n$ channel uses. The dashed lines represent interfering receptions. The dotted line represents the side-information path. The power constraints are $\widetilde{P}_{p}$ and $\widetilde{P}_{c}$ and noise variances are $N_{p}$ and $N_{s}$.

The additive noise at the primary and secondary receivers, $\widetilde{Z}_{p}^{n}:=\left(\widetilde{Z}_{p, 1}, \widetilde{Z}_{p, 2}, \ldots, \widetilde{Z}_{p, n}\right)$ and $\widetilde{Z}_{s}^{n}:=\left(\widetilde{Z}_{s, 1}, \widetilde{Z}_{s, 2}, \ldots, \widetilde{Z}_{s, n}\right)$, is assumed to be i.i.d. across symbol times $i=1,2, \ldots n$ and distributed according to $\mathcal{N}\left(0, N_{p}\right)$ and $\mathcal{N}\left(0, N_{s}\right)$, respectively ${ }^{3}$. The correlation be-

${ }^{3}$ Throughout the paper we will denote vectors in $\mathbb{R}^{n}$ by $X^{n}:=\left(X_{i}, X_{2}, \ldots, X_{n}\right)$ 
tween $\widetilde{Z}_{p}^{n}$ and $\widetilde{Z}_{s}^{n}$ is irrelevant from the standpoint of probability of error or capacity calculations since the base-stations are not allowed to pool their signals. The primary user has message $m_{p} \in\left\{0,1, \ldots, 2^{n R_{p}}\right\}$ intended for the primary receiver to decode, the cognitive user has message $m_{c} \in\left\{0,1, \ldots, 2^{n R_{c}}\right\}$ intended for the secondary receiver as well as the message $m_{p}$ of the primary user. The average power of the transmitted signals is constrained by $\widetilde{P}_{p}$ and $\widetilde{P}_{c}$, respectively:

$$
\left\|\widetilde{X}_{p}^{n}\right\|^{2} \leq n \widetilde{P}_{p}, \quad\left\|\widetilde{X}_{c}^{n}\right\|^{2} \leq n \widetilde{P}_{c}
$$

The received signal-to-noise ratios (SNRs) of the desired signals at the primary and secondary base-station are $p^{2} \widetilde{P}_{p} / N_{p}$ and $c^{2} \widetilde{P}_{c} / N_{s}$, respectively. The received SNRs of the interfering signals at the primary and secondary base-station (INRs) are $f^{2} \widetilde{P}_{c} / N_{p}$ and $g^{2} \widetilde{P}_{p} / N_{s}$, respectively. The constants $(p, c, f, g)$ are assumed to be real, positive and globally known. The results of this paper easily extend to the case of complex coefficients (see Section 5.3). The channel can be described by the pair of per-time-sample equations

$$
\begin{aligned}
& \tilde{Y}_{p}=p \widetilde{X}_{p}+f \widetilde{X}_{c}+\widetilde{Z}_{p} \\
& \widetilde{Y}_{s}=g \widetilde{X}_{p}+c \widetilde{X}_{c}+\widetilde{Z}_{s}
\end{aligned}
$$

where $\widetilde{Z}_{p}$ is $\mathcal{N}\left(0, N_{p}\right)$ and $\widetilde{Z}_{s}$ is $\mathcal{N}\left(0, N_{s}\right)$.

\subsection{Transformation to standard form}

We can convert every cognitive radio channel with gains $(p, f, g, c)$, power constraints $\left(\widetilde{P}_{p}, \widetilde{P}_{c}\right)$ and noise powers $\left(N_{p}, N_{s}\right)$ to a corresponding standard form cognitive radio channel with gains $(1, a, b, 1)$, power constraints $\left(P_{p}, P_{c}\right)$ and noise powers $(1,1)$, expressed by the pair of equations

$$
\begin{aligned}
& Y_{p}=X_{p}+a X_{c}+Z_{p}, \\
& Y_{s}=b X_{p}+X_{c}+Z_{s},
\end{aligned}
$$

where

$$
\begin{array}{ll}
a:=\frac{f \sqrt{N_{s}}}{c \sqrt{N_{p}}}, & b:=\frac{g \sqrt{N_{p}}}{p \sqrt{N_{s}}} \\
P_{p}:=\frac{p^{2} \widetilde{P}_{p}}{N_{p}}, & P_{c}:=\frac{c^{2} \widetilde{P}_{c}}{N_{s}} .
\end{array}
$$


The capacity of this cognitive radio channel is the same as that of the original channel since the two channels are related by invertible transformations ${ }^{4}$ that are given by

$$
\begin{aligned}
X_{p}:=\frac{p \widetilde{X}_{p}}{\sqrt{N_{p}}}, \quad Y_{p}:=\frac{\widetilde{Y}_{p}}{\sqrt{N_{p}}}, \quad Z_{p}:=\frac{\widetilde{Z}_{p}}{\sqrt{N_{p}}} ; \\
X_{c}:=\frac{c \widetilde{X}_{c}}{\sqrt{N_{s}}}, \quad Y_{s}:=\frac{\widetilde{Y}_{s}}{\sqrt{N_{s}}}, \quad Z_{s}:=\frac{\widetilde{Z}_{s}}{\sqrt{N_{s}}} .
\end{aligned}
$$

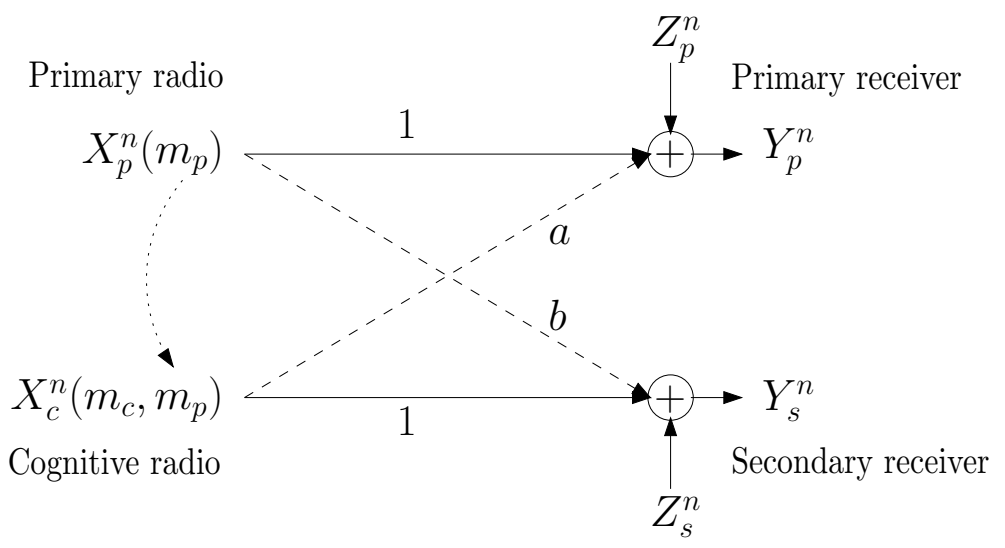

Figure 3: The cognitive radio channel in standard form. The channel gains $(p, f, g, c)$ in the original channel are mapped to $(1, a, b, 1)$, powers $\left(\widetilde{P}_{p}, \widetilde{P}_{c}\right)$ are mapped to $\left(P_{p}, P_{c}\right)$, and noise variances $\left(N_{p}, N_{s}\right)$ are mapped to $(1,1)$.

We will consider this standard form of the cognitive radio channel without loss of generality and we will refer to it as the $(1, a, b, 1)$ cognitive radio channel.

\subsection{Coding on the cognitive radio channel}

Let the channel input alphabets of the primary and cognitive radios be $\mathcal{X}_{p}=\mathbb{R}$ and $\mathcal{X}_{c}=\mathbb{R}$, respectively. Similarly, let the channel output alphabets at the primary and secondary receivers be $\mathcal{Y}_{p}=\mathbb{R}$ and $\mathcal{Y}_{s}=\mathbb{R}$, respectively.

The primary receiver is assumed to use a single-user decoder, $D_{p}^{n}: \mathcal{Y}_{p}^{n} \mapsto\left\{1,2, \ldots, 2^{n R_{p}}\right\}$, to decode $m_{p}$ from $Y_{p}^{n}$. We define a single-user decoder to be any decoder which performs well on the point-to-point AWGN channel. For concreteness, we set the primary decoder to be the nearest-neighbor (or minimum-distance) decoder. Hence, we have the following definition.

\footnotetext{
${ }^{4}$ These transformations were used in [1], [3] and [18], in the context of the classical interference channel.
} 
Definition 2.1 (Achievability: primary user) $A$ rate $R_{p}$ is achievable for the primary user if there exists a sequence of encoders $E_{p}^{n}:\left\{1,2, \ldots, 2^{n R_{p}}\right\} \mapsto \mathcal{X}_{p}^{n}$ such that the average probability of error ${ }^{5}$ vanishes as $n \rightarrow \infty$, i.e.,

$$
P_{e, p}^{(n)} \stackrel{\text { def }}{=} \frac{1}{2^{n R_{p}}} \sum_{i=1}^{2^{n R_{p}}} \mathbb{P}\left(D_{p}^{n}\left(Y_{p}^{n}\right) \neq i \mid m_{p}=i\right) \rightarrow 0,
$$

when the primary receiver uses a single-user decoder, $D_{p}^{n}$.

The salient feature of the cognitive radio is that it has knowledge of the primary encoder as well as the message $m_{p}$. Let $\mathcal{E}_{p}^{n}$ denote the set of all primary encoders. Hence, we have the following definition:

Definition 2.2 (Cognitive radio code) $A\left(2^{n R_{c}}, n\right)$ cognitive radio code is a choice of an encoding rule (whose output we denote by $X_{c}^{n}$ )

$$
E_{c}^{n}: \mathcal{E}_{p}^{n} \times\left\{1,2, \ldots, 2^{n R_{p}}\right\} \times\left\{1,2, \ldots, 2^{n R_{c}}\right\} \rightarrow \mathcal{X}_{c}^{n},
$$

such that $\left\|X_{c}^{n}\right\|^{2} \leq n P_{c}$, and a choice of a decoding rule

$$
D_{c}^{n}: \mathcal{Y}_{s}^{n} \rightarrow\left\{1,2, \ldots, 2^{n R_{c}}\right\}
$$

The following key definition formalizes the important notion of coexistence conditions that the cognitive secondary system must satisfy.

Definition 2.3 (Achievability: cognitive user) $A$ rate $R_{c}$ is said to be achievable for the cognitive user on $a(1, a, b, 1)$ cognitive radio channel if there exists a sequence of $\left(2^{n R_{c}}, n\right)$ cognitive radio codes such that the following two constraints are satisfied:

1. The average probability of error vanishes as $n \rightarrow \infty$, i.e.,

$$
P_{e, c}^{(n)} \stackrel{\text { def }}{=} \frac{1}{2^{n\left(R_{c}+R_{p}\right)}} \sum_{i=1, j=1}^{n} \mathbb{P}\left(D_{c}^{n}\left(Y_{s}^{n}\right) \neq j \mid m_{p}=i, m_{c}=j\right) \rightarrow 0 ;
$$

2. A rate of $R_{p}^{*} \stackrel{\text { def }}{=} \frac{1}{2} \log \left(1+P_{p}\right)$ is achievable for the primary user, in the sense of Definition 2.1.

We note that the above definition is well-defined, since the rate $R_{c}=0$ is achievable for the cognitive user if the cognitive radio simply shuts off.

Definition 2.4 (Capacity) The capacity of the cognitive radio channel is defined to be the largest achievable rate $R_{c}$ for the cognitive user.

\footnotetext{
${ }^{5}$ The tuple $\left(m_{p}, m_{c}\right)$ is assumed to be drawn from a uniform product measure on $\left\{1,2, \ldots, 2^{n R_{p}}\right\} \times$ $\left\{1,2, \ldots, 2^{n R_{c}}\right\}$.
} 


\section{The Capacity of the Cognitive Radio Channel}

If the received SNR of the cognitive radio transmission is lesser at the primary receiver than at the secondary receiver, we say that the primary system is affected by a low interference gain. This is the case that is most likely to occur in practice since the cognitive radio is typically closer to its intended receiver (the secondary base-station) than to the primary base-station. In terms of the parameters of our problem, this situation corresponds to $f \sqrt{N_{s}} \leq c \sqrt{N_{p}}$ in our original cognitive radio channel, or, equivalently, to $a \leq 1$ in the corresponding standard-form $(1, a, b, 1)$ cognitive radio channel. The capacity of the cognitive radio channel in this regime is given by the following expression.

Theorem 3.1 The capacity of the $(1, a, b, 1)$ cognitive radio channel is

$$
R_{c}^{*}=\frac{1}{2} \log \left(1+\left(1-\alpha^{*}\right) P_{c}\right)
$$

as long as $a \leq 1$. The constant $\alpha^{*} \in[0,1]$ is given by

$$
\alpha^{*}=\left(\frac{\sqrt{P_{p}}\left(\sqrt{1+a^{2} P_{c}\left(1+P_{p}\right)}-1\right)}{a \sqrt{P_{c}}\left(1+P_{p}\right)}\right)^{2} \text {. }
$$

Note that Theorem 3.1 holds for any $b \in \mathbb{R}$ (or equivalently any $p, g \in \mathbb{R}$ in the original cognitive radio channel).

\subsection{Proof of the forward part}

To show the existence of a capacity-achieving cognitive $\left(2^{n R_{c}^{*}}, n\right)$ code, we generate a sequence of random codes such that the average probability of error (averaged over the ensemble of codes and messages) vanishes as $n \rightarrow \infty$. In particular, we have the following encoders and decoders:

- $E_{p}^{n}$ ensemble: Given $m_{p} \in\left\{1,2, \ldots, 2^{n R_{p}}\right\}$, generate the codeword $X_{p}^{n} \in \mathbb{R}^{n}$ by drawing its coordinates i.i.d. according to $\mathcal{N}\left(0, P_{p}\right)$.

- $E_{c}^{n}$ ensemble: Since the cognitive radio knows $m_{p}$ as well as $E_{p}^{n}$, it can form $X_{p}^{n}$ and perform superposition coding as follows:

$$
X_{c}^{n}=\hat{X}_{c}^{n}+\sqrt{\frac{\alpha P_{c}}{P_{p}}} X_{p}^{n}
$$


where $\alpha \in[0,1]$. The codeword $\hat{X}_{c}^{n}$ encodes $m_{c} \in\left\{1,2, \ldots, 2^{n R_{c}}\right\}$ and is generated by performing Costa precoding [3] (also known as dirty-paper coding) treating $(b+$ $\left.\sqrt{\alpha \frac{P_{c}}{P_{p}}}\right) X_{p}^{n}$ as non-causally known interference that will affect the secondary receiver in the presence of $\mathcal{N}(0,1)$ noise. The encoding is done by random binning [3].

- $D_{p}^{n}$ : Single-user decoder that is optimal for the point-to-point AWGN channel, such as the nearest-neighbor decoder.

- $D_{c}^{n}$ : Costa decoder (having knowledge of the binning encoder $E_{c}^{n}$ ) [3].

The key result of Costa [4] is that, using the dirty-paper coding technique, the maximum achievable rate is the same as if the interference was also known at the receiver, i.e., as if it were absent altogether. The characteristic feature of this scheme is that the resulting codeword $\hat{X}_{c}^{n}$ is statistically independent of $X_{p}^{n}$ and is i.i.d. Gaussian. To satisfy the average power constraint of $P_{c}$ on the components of $X_{c}^{n}$, each coordinate of $\hat{X}_{c}^{n}$ must, in fact, be $\mathcal{N}\left(0,(1-\alpha) P_{c}\right)$. Hence, the primary receiver can treat $\hat{X}_{c}^{n}$ as independent Gaussian noise. Using standard methodology, it can be shown that the average probability of error for decoding $m_{p}$ (averaged over the code ensembles and messages) vanishes, as $n \rightarrow \infty$, for all rates $R_{p}$ below

$$
\frac{1}{2} \log \left(1+\frac{\left(\sqrt{P_{p}}+a \sqrt{\alpha P_{c}}\right)^{2}}{1+a^{2}(1-\alpha) P_{c}}\right) .
$$

Similarly, the average probability of error in decoding $m_{c}$ vanishes for all rates $R_{c}$ below

$$
\frac{1}{2} \log \left(1+(1-\alpha) P_{c}\right)
$$

However, in order to ensure that a given rate is achievable for the cognitive user in the sense of Definition 2.3, we must have that

$$
\frac{1}{2} \log \left(1+\frac{\left(\sqrt{P_{p}}+a \sqrt{\alpha P_{c}}\right)^{2}}{1+a^{2}(1-\alpha) P_{c}}\right)=\frac{1}{2} \log \left(1+P_{p}\right)=: R_{p}^{*} .
$$

Observe that, if $a=0$, any choice of $\alpha \in[0,1]$ will satisfy (18): in this case we should set $\alpha^{*}=0$ to maximize the rate achievable for the cognitive user. For $a>0$, by the Intermediate Value Theorem, this quadratic equation in $\alpha$ always has a unique root in $[0,1]$, and it is given by $(14)$.

Finally, since the code-ensemble-averaged (and message-averaged) probabilities of error vanish, there must exist a particular sequence of cognitive radio codes and primary encoders for which the (message-averaged) probabilities of error vanish as well. Hence, $R_{c}^{*}=\frac{1}{2} \log \left(1+\left(1-\alpha^{*}\right) P_{c}\right)$ is achievable for the cognitive user in the sense of Definition 2.3. 


\subsection{Proof of the converse part}

\subsubsection{Proof outline}

We will first relax the constraints of our problem and allow for joint primary and cognitive radio code design thus forming an interference channel with degraded message sets ${ }^{6}$, which we will abbreviate as IC-DMS for convenience. Since this relaxation enlarges the space of allowable encoder-decoder pairs, the largest set of achievable rate pairs $\left(R_{p}, R_{c}\right)$ in the IC-DMS must include the rate point corresponding to the capacity of the cognitive radio channel, i.e., $\left(\frac{1}{2} \log \left(1+P_{p}\right), R_{c}^{*}\right)$, whatever $R_{c}^{*}$ might be.

Our approach is to first characterize the capacity region of the IC-DMS, i.e., the largest set of rate tuples $\left(R_{p}, R_{c}\right)$ at which joint reliable communication can take place. We are able to do this only for a part of the capacity region ${ }^{7}$ and we refer the reader to a concurrent and independent work [24] which provides a proof for the entire capacity region. Next we make the key observation that the joint coding scheme that achieves all the rate tuples in the capacity region of the IC-DMS has the property that the decoder at the primary receiver is a standard single-user decoder. Furthermore, we show that largest value of $R_{c}$ such that the point $\left(R_{p}, R_{c}\right)=\left(\frac{1}{2} \log \left(1+P_{p}\right), R_{c}\right)$ is on the boundary of the capacity region of the IC-DMS is given by $R_{c}^{*}=\frac{1}{2} \log \left(1+\left(1-\alpha^{*}\right) P_{c}\right)$ with $\alpha^{*}$ as in (14). We then conclude that $R_{c}=R_{c}^{*}$ is the capacity of the corresponding cognitive radio channel.

\subsubsection{Joint code design: The IC-DMS}

The input-output equations of the IC-DMS, as for the cognitive radio channel, are given by (2), (3) with the standard form given by (4), (5). We will denote the IC-DMS in standard form by "( $1, a, b, 1)$-IC-DMS".

Definition 3.1 (IC-DMS code) $A\left(2^{n R_{p}}, 2^{n R_{c}}, n\right)$ code for the $(1, a, b, 1)-I C-D M S$ is a joint selection of the encoding rules and the decoding rules: the encoding rules are two maps (whose outputs we denote by $X_{p}^{n}$ and $X_{c}^{n}$, respectively), denoted as

$$
\begin{array}{ll}
e_{p}^{n}: & \left\{1,2, \ldots, 2^{n R_{p}}\right\} \rightarrow \mathcal{X}_{p}^{n}, \\
e_{c}^{n}: & \left\{1,2, \ldots, 2^{n R_{p}}\right\} \times\left\{1,2, \ldots, 2^{n R_{c}}\right\} \rightarrow \mathcal{X}_{c}^{n},
\end{array}
$$

\footnotetext{
${ }^{6}$ The primary user knows $m_{p}$ while the cognitive user knows $\left\{m_{p}, m_{c}\right\}$, hence the primary user has a subset of the messages available to the cognitive user.

${ }^{7}$ The original version of this paper had an error in the proof of the converse for the complementary portion of the capacity region.
} 
such that $\left\|X_{p}^{n}\right\|^{2} \leq n P_{p}$ and $\left\|X_{c}^{n}\right\|^{2} \leq n P_{c}$. The decoding rules are two maps denoted as

$$
\begin{aligned}
d_{p}^{n}: & \mathcal{Y}_{p}^{n} \rightarrow\left\{1,2, \ldots, 2^{n R_{p}}\right\}, \\
d_{c}^{n}: & \mathcal{Y}_{s}^{n} \rightarrow\left\{1,2, \ldots, 2^{n R_{c}}\right\} .
\end{aligned}
$$

Given that the messages selected are $\left(m_{p}=i, m_{c}=j\right)$, an error occurs if $d_{p}^{n}\left(Y_{p}^{n}\right) \neq i$ or $d_{c}^{n}\left(Y_{s}^{n}\right) \neq j$.

Observe that, unlike in the original cognitive radio channel, here we allow for an arbitrary choice of the primary decoder and, in particular, for the possibility of multiuser decoding, an example of which is successive interference decoding and cancelation.

Definition 3.2 (Achievability: IC-DMS) A rate vector $\left(R_{p}, R_{c}\right)$ is said to be achievable if there exists a sequence of $\left(2^{n R_{p}}, 2^{n R_{c}}, n\right)$ codes such that the average probability of error at each of the receivers vanishes as $n \rightarrow \infty$, i.e.,

$$
\begin{aligned}
& \widetilde{P}_{e, p}^{(n)} \stackrel{\text { def }}{=} \frac{1}{2^{n\left(R_{c}+R_{p}\right)}} \sum_{i=1, j=1}^{n} \mathbb{P}\left(d_{p}^{n}\left(Y_{p}^{n}\right) \neq i \mid m_{p}=i, m_{c}=j\right) \rightarrow 0, \\
& P_{e, c}^{(n)} \stackrel{\text { def }}{=} \frac{1}{2^{n\left(R_{c}+R_{p}\right)}} \sum_{i=1, j=1}^{n} \mathbb{P}\left(d_{c}^{n}\left(Y_{s}^{n}\right) \neq j \mid m_{p}=i, m_{c}=j\right) \rightarrow 0 .
\end{aligned}
$$

Definition 3.3 (Capacity region) The capacity region of the IC-DMS is the closure of the set of achievable rate vectors $\left(R_{p}, R_{c}\right)$.

\subsubsection{The capacity region of the IC-DMS under a low interference gain}

The following theorem characterizes a part of the capacity region of the $(1, a, b, 1)$-ICDMS with $a \leq 1$ and arbitrary $b \in \mathbb{R}$. As mentioned earlier, our result here is subsumed by the result independently and concurrently obtained in [24], Theorem 3.5.

Theorem 3.2 The segment of the boundary, corresponding to $\frac{d R_{c}}{d R_{p}} \geq-1$, of the capacity region of the $(1, a, b, 1)-I C$-DMS with $a \leq 1$ and $b \in \mathbb{R}$ is given by

$$
\begin{aligned}
& R_{p}(\alpha)=\frac{1}{2} \log \left(1+\frac{\left(\sqrt{P_{p}}+a \sqrt{\alpha P_{c}}\right)^{2}}{1+a^{2}(1-\alpha) P_{c}}\right) \\
& R_{c}(\alpha)=\frac{1}{2} \log \left(1+(1-\alpha) P_{c}\right),
\end{aligned}
$$

where $\alpha \in[0,1]$ satisfies the condition $\frac{d R_{c}(\alpha)}{d R_{p}(\alpha)} \geq-1$. 
Proof of achievability: The random coding scheme described in the forward part of the proof of Theorem 3.1 (Section 3.1) achieves the rates (25) and (26) stated in the theorem. We emphasize that, in this scheme, the primary receiver employs a single-user decoder.

Proof of converse: See Appendix A.

The result of [24], Theorem 3.5, further proves that the segment of the boundary of the capacity region complementary to the one stated above, i.e., the segment satisfying

$\frac{d R_{c}}{d R_{p}}<-1$, is also given by the equations (25) and (26). Thus, the entire capacity region of the low-interference IC-DMS is obtained ${ }^{8}$.

\subsubsection{The capacity of the cognitive radio channel under a low interference gain}

The proof of Theorem 3.2 reveals that the jointly designed code that achieves all the points on the boundary of the capacity region of the IC-DMS is such that the primary receiver uses a standard single-user decoder, just as it would in the absence of the cognitive radio. In other words, the primary decoder $d_{p}^{n}$ does not depend on $e_{c}^{n}$ and $d_{c}^{n}$. Thus, in order to find the largest rate that is achievable by the cognitive user in the sense of Definition 2.3 we can without loss of generality restrict our search to the boundary of the capacity region of the underlying IC-DMS. Hence, to find this capacity of the cognitive radio channel, we must solve for the positive root of the quadratic equation (18) in $\alpha$. The solution is given by $\alpha^{*}$ in (14), hence the capacity is

$$
R_{c}^{*}=\frac{1}{2} \log \left(1+\left(1-\alpha^{*}\right) P_{c}\right) .
$$

Thus we have established the proof of Theorem 3.1.

\section{The High-interference-gain Regime}

The technique used to prove the converse of Theorem 3.2 also allows us to characterize the sum-capacity of the $(1, a, b, 1)$-IC-DMS for any $a \geq 1$ and $b \in \mathbb{R}$, and the entire capacity region if $a$ is sufficiently large and $b$ is small enough. These two ancillary results are presented in this section.

\footnotetext{
${ }^{8}$ The original version of our paper contained an upper bound for the $\frac{d R_{c}}{d R_{p}}<-1$ portion of the boundary as well, but was found to not be tight, in general. Thanks to Hesham El Gamal for pointing this out to us.
} 


\subsection{The sum-capacity for $a \geq 1$}

Corollary 4.1 The maximum of $R_{p}+R_{c}$ over all $\left(R_{p}, R_{c}\right)$ in the capacity region of the $(1, a, b, 1)-I C$-DMS with $a \geq 1$ and $b \in \mathbb{R}$ is achieved with $\alpha=1$ in (25) and (26), i.e.,

$$
C_{\text {sum }}(a)=\frac{1}{2} \log \left(1+\left(\sqrt{P_{p}}+a \sqrt{P_{c}}\right)^{2}\right) .
$$

Proof: See Appendix B

\subsection{The benefit of joint code design}

We emphasize that the scheme that is optimal in the low-interference gain regime has the property that the primary receiver employs a single-user decoder. Contrary to this, we now observe that, when $a$ is large enough, the optimal (jointly designed) IC-DMS code is such that the primary decoder $d_{p}^{n}$ depends on the cognitive encoder $e_{c}^{n}$. First, we demonstrate an achievable scheme in the following lemma.

Lemma 4.1 Consider the $(1, a, b, 1)-I C$-DMS. For every $\alpha \in[0,1]$, the rate pair $\left(R_{p}, R_{c}\right)$ satisfying

$$
\begin{aligned}
& R_{p}=\hat{R}_{p}(\alpha) \stackrel{\text { def }}{=} \frac{1}{2} \log \left(1+\left(\sqrt{P_{p}}+a \sqrt{\alpha P_{c}}\right)^{2}\right) \\
& R_{c}=\hat{R}_{c}(\alpha) \stackrel{\text { def }}{=} \frac{1}{2} \log \left(1+\frac{(1-\alpha) P_{c}}{1+\left(b \sqrt{P_{p}}+\sqrt{\alpha P_{c}}\right)^{2}}\right),
\end{aligned}
$$

is achievable as long as

$$
a \geq \frac{\sqrt{\alpha P_{p} P_{c}}}{K(\alpha)}+\sqrt{K(\alpha)+P_{p}\left(1+\left(b \sqrt{P_{p}}+\sqrt{\alpha P_{c}}\right)^{2}\right)},
$$

where $K(\alpha) \stackrel{\text { def }}{=} 1+b^{2} P_{p}+2 b \sqrt{\alpha P_{p} P_{c}}$.

Proof: The primary transmitter forms $X_{p}^{n}$ by drawing its coordinates i.i.d. according to $\mathcal{N}\left(0, P_{p}\right)$. Since the cognitive radio knows $m_{p}$ and $e_{p}^{n}$ it forms $X_{p}^{n}$ then generates $X_{c}^{n}$ by superposition coding:

$$
X_{c}^{n}=\hat{X}_{c}^{n}+\sqrt{\frac{\alpha P_{c}}{P_{p}}} X_{p}^{n}
$$


where $\hat{X}_{c}^{n}$ is formed by drawing its coordinates i.i.d. according to $\mathcal{N}\left(0, \sqrt{(1-\alpha) P_{c}}\right)$ for some $\alpha \in[0,1]$. The decoder $d_{p}^{n}$ at the primary receiver first decodes $m_{c}$ treating $\left(1+a \sqrt{\alpha P_{c} / P_{p}}\right) X_{p}^{n}$ as independent Gaussian noise. It then reconstructs $a \hat{X}_{c}^{n}$ (which it can do because it knows $e_{c}^{n}$ ) and subtracts off its contribution from $Y_{p}^{n}$ before decoding $m_{p}$. The decoding rule $d_{c}^{n}$ at the secondary receiver is simply to decode $m_{c}$ treating $\left(b+\sqrt{\alpha P_{c} / P_{p}}\right) X_{p}^{n}$ as independent Gaussian noise. The rates achievable with this scheme are then exactly given by (29) and (30), provided that the rate at which the primary receiver can decode the cognitive user's message is not the limiting factor, i.e.,

$$
\frac{(1-\alpha) P_{c}}{1+\left(b \sqrt{P_{p}}+\sqrt{\alpha P_{c}}\right)^{2}} \leq \frac{a^{2}(1-\alpha) P_{c}}{1+\left(\sqrt{P_{p}}+a \sqrt{\alpha P_{c}}\right)^{2}} .
$$

Solving this quadratic inequality for $a$, we find that the condition is satisfied only when $a$ satisfies inequality (31) stated in the theorem.

In the achievable scheme described in Lemma 4.1, the primary receiver decodes the message of the cognitive user before decoding the message of the primary user. This scheme, in fact, is optimal for the $(1, a, b, 1)$-IC-DMS when $a$ is sufficiently large and $b$ is small enough. This statement is made precise in the following theorem.

Theorem 4.2 A point $\left(R_{p}, R_{c}\right)$ is on the boundary of the capacity region of the $(1, a, b, 1)$ $I C$-DMS if there exists $\alpha \in[0,1]$ such that

1. $\left(R_{p}, R_{c}\right)=\left(\hat{R}_{p}(\alpha), \hat{R}_{c}(\alpha)\right)$ where $\hat{R}_{p}(\alpha)$ and $\hat{R}_{c}(\alpha)$ are defined in $(29)$ and $(30)$, respectively,

2. $a$ and $b$ satisfy the condition given in (31), and

3. $b \leq b_{\max }\left(\mu_{\alpha}, a\right)$ where $\mu_{\alpha} \stackrel{\text { def }}{=}-\left.\frac{d^{-} \hat{R}_{c}(x)}{d \hat{R}_{p}(x)}\right|_{x=\alpha}$ and $b_{\max }(\mu, a)$ is defined in Appendix $C$.

Proof of achievability: Given in Lemma 4.1.

Proof of converse: Given in Appendix C.

As $a$ gets larger and $b$ gets smaller, Theorem 4.2 captures a larger portion of the boundary of the capacity region, until it finally characterizes the entire capacity region when $a \geq \sqrt{P_{p} P_{c}} / K(1)+\sqrt{K(1)+P_{p}\left(1+\left(b \sqrt{P_{p}}+\sqrt{P_{c}}\right)^{2}\right)}$ and $b \leq b_{\max }\left(\mu_{1}, a\right)$.

\section{System-level Considerations}

In this section we use our results on the capacity-achieving cognitive communication scheme to derive insight into a practical implementation of cognitive radios. 


\subsection{Properties of the optimal scheme}

\subsubsection{Avoiding the "hidden-terminal" problem}

The network of Fig. 1 models the situation in which the geographic location of $B_{s}$ is not assigned in accordance with any centralized cell-planning policy and it can be arbitrarily close to $B_{p}$. Consequently, the secondary users that are in close proximity to $B_{p}$ could potentially cause significant interference for the primary system if the secondary system is to operate over the same frequency band.

One possible adaptive communication scheme that the cognitive radio could employ in order to avoid interfering with the primary user in its vicinity would be to restrict its transmissions to only the time-frequency slots which are not occupied by the signals of the detected primary radio. Indeed, this idea of "opportunistic" orthogonal communication was what led to the birth of the notion of cognitive radio. However, one drawback of such a protocol is that the cognitive radio would very likely cause interference to other, more distant, primary users whose presence - i.e., time-frequency locations - it could not detect. The degradation in overall performance of the primary system due to this "hidden-terminal" problem could potentially be significant ${ }^{9}$, especially in the context of OFDMA [9], [10] where the primary users are allocated orthogonal time-frequency slots and the SINR required for decoding is typically large.

Contrary to this, we find that the optimal strategy is for the cognitive radio to simultaneously transmit in the same frequency slot as that used by the primary user in its vicinity. An immediate benefit of this scheme is that, if the transmissions of different primary users are mutually orthogonal, the cognitive radio can only (potentially) affect the performance achievable by the primary radio whose codeword it has decoded. Furthermore, we know that a proper tuning of the parameter $\alpha$ can, in fact, ensure that the primary user's rate is unaffected.

\subsubsection{Robustness to noise statistics}

All our results have been derived under the assumption that the noise affecting the receivers, $Z_{p}^{n}$ and $Z_{s}^{n}$, is i.i.d. Gaussian. In [2] it was shown that using a Costa encoderdecoder pair that is designed for additive i.i.d Gaussian noise on a channel with arbitrary (additive) noise statistics will cause no loss in the achievable rates. ${ }^{10}$ Combined with the similar classical result for the standard AWGN channel [12], we see that the maximal rate expressed in Theorem 3.1 is achievable for all noise distributions.

\footnotetext{
${ }^{9}$ Classical RTS/CTS solutions to this problem are not viable since they require that the primary system ask for access to the very spectrum that it owns.

${ }^{10}$ Note that this is an achievability result: the capacity of the channel with this arbitrary noise could be larger but a different code would be required to achieve it.
} 


\subsection{Obtaining the side-information}

In practice, the cognitive radio is limited to obtaining the primary radio's codeword in a causal fashion - its acquisition thus introducing delays in the cognitive radio transmissions ${ }^{11}$. In a typical situation, due to its relative proximity to the primary user, the cognitive radio can receive the primary transmissions with a greater received SNR than that experienced by the primary receiver. Hence, it seems plausible that the cognitive radio could decode ${ }^{12}$ the message of the primary user in fewer channel uses than are required by the primary receiver. Recent work in distributed space-time code design [15] indicates that this overhead decoding delay is negligible if the cognitive radio has as little as a $10 \mathrm{~dB}$ advantage in the received SNR over the primary receiver.

In a practical implementation of a secondary system, the cognitive radio could be designed to efficiently exploit the the Automatic Repeat Request (ARQ) mechanism employed by the primary system. Most wireless systems today utilize ARQ protocols to increase the uplink packet decoding reliability: the retransmissions are either identical repeats of the erroneously decoded data frame (standard ARQ) or a subset of the parity bits of a punctured systematic error-correcting code (Incremental Redundancy Hybrid$A R Q$ ). In the presence of an ARQ protocol, the first transmission of the primary radio is usually under-powered (to minimize the energy consumption) and the primary basestation is likely to send back a NAK. However, due to its proximity to the primary radio, the cognitive radio is very likely to have successfully decoded the data bits and could then communicate to its receiver during the next ARQ round(s) using the optimal code without the need to again listen and decode the primary transmission.

The key assumption here is that the cognitive radio is able to decode the ACK/NAK signals from the primary base-station. Also, the cognitive radio must know the ARQ scheme being used (the particular code, puncturing pattern and power increments in the case of IR Hybrid-ARQ). This information is typically periodically broadcast in the downlink of cellular systems for the benefit of new-coming primary users and a cognitive radio that is synchronized to the primary system could also extract this information.

\subsection{Extension to complex baseband}

The results of this paper can easily be extended to the case in which the channel gains are complex quantities, i.e., $p, f, g, c \in \mathbb{C}$ in the case of the original $(p, f, g, c)$ cognitive radio channel with power constraints $\left(P_{p}, P_{c}\right)$ and noise variances $\left(N_{p}, N_{s}\right)$, as defined in Section 2.1. However, the optimal cognitive encoder rule (15) must change slightly: The

\footnotetext{
${ }^{11}$ Under a half-duplex constraint the cognitive radio must first "listen" in order to decode the primary message before it can use this side-information for its own transmission.

${ }^{12}$ The cognitive radio is assumed to know the encoder of the primary user.
} 
superposition scheme takes the form

$$
X_{c}^{n}=\hat{X}_{c}^{n}+\frac{f^{*}}{|f|} e^{j \theta_{p}} \sqrt{\alpha \frac{P_{c}}{P_{p}}} X_{p}^{n},
$$

where $p=|p| e^{j \theta_{p}}$. The codeword $\hat{X}_{c}^{n}$ is again generated by Costa precoding, but the assumed interference at the secondary receiver is now

$$
\left(\frac{g}{c}+\frac{f^{*}}{|f|} e^{j \theta_{p}} \sqrt{\alpha \frac{P_{c}}{P_{p}}}\right) X_{p}^{n}
$$

and the assumed noise is $\mathcal{C N}\left(0, N_{s} /|c|^{2}\right)$. The factor $e^{j \theta_{p}}$ in (32) essentially implements transmit beamforming to the primary receiver, hence ensuring that all the rates given by

$$
\begin{aligned}
& 0 \leq R_{p} \leq \log \left(1+\frac{\left(|p| \sqrt{P_{p}}+|f| \sqrt{\alpha P_{c}}\right)^{2}}{N_{p}+|f|^{2}(1-\alpha) P_{c}}\right) \\
& 0 \leq R_{c} \leq \log \left(1+\frac{|c|^{2}(1-\alpha) P_{c}}{N_{s}}\right)
\end{aligned}
$$

are achieved in the underlying IC-DMS. As before, we can then choose $\alpha=\alpha^{*}$ (determined by (14)), so that $R_{c}^{*}=\log \left(1+|c|^{2}\left(1-\alpha^{*}\right) P_{c} / N_{s}\right)$ is achievable in the spirit of Definition 2.3 but with $R_{p}^{*}=\log \left(1+|p|^{2} P_{p} / N_{p}\right)$.

\subsection{Communicating without channel-state feedback from the primary base-station}

In order to perform the complex base-band superposition coding scheme (32) and, implicitly, the Costa precoding for known interference (33), the cognitive radio must know each of the four parameters $g, c, f$ and $p$, both in magnitude and phase.

To obtain estimates for both the magnitude and phase of $p$ and $f$, the cognitive radio would require additional help from the primary system in the form of channelstate feedback from the primary base-station. In Section 5.5, we present a method for accomplishing this, based on the assumption that the cognitive radio can extract the channel-state feedback intended for the primary radio. In this section, however, we present an alternative scheme which requires no feedback from the primary base-station and which achieves the low-SNR capacity of a "fast-fading" cognitive radio channel under the assumption that the cognitive radio has no knowledge of $p$ and $f$. 


\subsubsection{The capacity in the absence of channel state information}

Suppose that, after having decoded $X_{p}^{n}$, the cognitive radio transmits the following $n$ symbol codeword:

$$
X_{c}^{n}=\hat{X}_{c}^{n}+\sqrt{\alpha \frac{P_{c}}{P_{p}}} X_{p}^{n},
$$

where the codeword $\hat{X}_{c}^{n}$ is generated by Costa precoding for the interference

$$
\left(\frac{g}{c}+\sqrt{\alpha \frac{P_{c}}{P_{p}}}\right) X_{p}^{n},
$$

assuming the presence of $\mathcal{C N}\left(0, N_{s} /|c|^{2}\right)$ noise at the secondary base-station. Note that this scheme does not require knowledge of the channel between the cognitive radio and the primary receiver, parameter $f$, or the channel between the primary radio and primary reciever, parameter $p .{ }^{13}$ The cognitive radio does, however, require knowledge of the channel to its receiver, parameter $c$, and the channel between the primary radio and the secondary receiver, parameter $g$ :

- Obtaining c: The parameter $c$ could be estimated at the secondary base-station by using the cognitive radio's pilot signal or in a decision-directed fashion. The estimate could then be fed back to the cognitive radio.

- Obtaining g: If the secondary base-station synchronizes to the primary radio's pilot signal, it could estimate $g$ during the time the cognitive radio is in its silent "listening" phase and then feed this estimate back to the cognitive radio. Alternatively, if the cognitive radio reveals to the secondary base-station the code used by the primary radio, the secondary base-station could use the silent "listening" phase to decode a few symbols transmitted by the primary radio thereby estimating the parameter $g$.

This cognitive communication scheme is, in fact, optimal for the IC-DMS channel in which $p$ and $f$ are not known at the cognitive (and primary) radio, but are known at the primary receiver and are fixed for the duration of communication. In this case, it is straightforward to see that the boundary of the capacity region of this IC-DMS, parametrized by $\alpha \in[0,1]$, is given by

$$
\begin{aligned}
& R_{p}=\log \left(1+\frac{\left|p \sqrt{P_{p}}+f \sqrt{\alpha P_{c}}\right|^{2}}{N_{p}+|f|^{2}(1-\alpha) P_{c}}\right), \\
& R_{c}=\log \left(1+\frac{|c|^{2}(1-\alpha) P_{c}}{N_{s}}\right) .
\end{aligned}
$$

\footnotetext{
${ }^{13}$ Note that the parameter $\alpha$ in (36) could potentially depend on $p$ or $f$. As it will turn out, its "optimal" value depends only on the magnitude of $p$, as will be shown in eq. (42).
} 
Observe that since $p, f \in \mathbb{C}$, the quantity $\left|p \sqrt{P_{p}}+f \sqrt{\alpha P_{c}}\right|^{2}$ could be arbitrarily small implying that the only way that the cognitive radio can ensure that it does not interfere with the primary radio is to completely shut off. In other words, the capacity, in the sense of Definition 2.4, of the cognitive radio channel is zero when $p$ and $f$ are not known at the cognitive radio and are fixed for the duration of communication.

However, such an extreme slow fading scenario is unlikely in practice: a more reasonable assumption might be that $p$ and $f$ are time varying (and still tracked at the primary receiver). Under the assumption of ideal interleaving, we can model these channel states $\{p[m]\}$ and $\{f[m]\}$ as ergodic random processes. In this case, the boundary of the capacity region of this "fast-fading" IC-DMS is given by:

$$
\begin{aligned}
& R_{p}=\mathbb{E}\left[\log \left(1+\frac{\left|p \sqrt{P_{p}}+f \sqrt{\alpha P_{c}}\right|^{2}}{N_{p}+|f|^{2}(1-\alpha) P_{c}}\right)\right], \\
& R_{c}=\log \left(1+\frac{|c|^{2}(1-\alpha) P_{c}}{N_{s}}\right),
\end{aligned}
$$

where the expectation is taken with respect to the stationary distribution of the ergodic processes. If we further assume that $p$ and $f$ are independent and their magnitude are deterministic, we can obtain the following low-SNR order approximation ${ }^{14}$ for (38):

$$
R_{p}=\Theta\left(\frac{|p|^{2} P_{p}+|f|^{2} \alpha P_{c}}{N_{p}+|f|^{2}(1-\alpha) P_{c}}\right), \quad \text { as } \quad \max \left\{\frac{|p|^{2} P_{p}}{N_{p}}, \frac{|f|^{2} P_{c}}{N_{p}}\right\} \rightarrow 0
$$

On the other hand, the capacity, $R_{p}^{*}$, of the point-to-point channel from the primary radio to its receiver, in the absence of the cognitive radio, satisfies

$$
R_{p}^{*} \stackrel{\text { def }}{=} \log \left(1+\frac{|p|^{2} P_{p}}{N_{p}}\right)=\Theta\left(\frac{|p|^{2} P_{p}}{N_{p}}\right), \quad \text { as } \quad \frac{|p|^{2} P_{p}}{N_{p}} \rightarrow 0
$$

Hence, in order to avoid causing interference to the primary user at low SNR, the following equation must be satisfied:

$$
\frac{|p|^{2} P_{p}+|f|^{2} \alpha P_{c}}{N_{p}+|f|^{2}(1-\alpha) P_{c}}=\frac{|p|^{2} P_{p}}{N_{p}}
$$

If the cognitive radio tunes its parameter $\alpha$ such that

$$
\alpha=\alpha_{*} \stackrel{\text { def }}{=} \frac{|p|^{2} P_{p} / N_{p}}{1+|p|^{2} P_{p} / N_{p}},
$$

\footnotetext{
${ }^{14}$ We say that $f(x)=\Theta(g(x))$ as $x \rightarrow 0$ if there exist constants $K_{1}, K_{2}>0$ such that $\lim _{x \rightarrow 0} \frac{f(x)}{g(x)} \leq K_{1}$ and $\lim _{x \rightarrow 0} \frac{f(x)}{g(x)} \geq K_{2}$.
} 
this condition will be satisfied, hence $R_{p}=\Theta\left(\frac{|p|^{2} P_{p}}{N_{p}}\right)$. The capacity of this "fast-fading" channel is then given by

$$
R_{c}=\log \left(1+\frac{|c|^{2}\left(1-\alpha_{*}\right) P_{c}}{N_{s}}\right), \quad \text { as } \quad \max \left\{\frac{|p|^{2} P_{p}}{N_{p}}, \frac{|f|^{2} P_{c}}{N_{p}}\right\} \rightarrow 0 .
$$

In order to compute $\alpha_{*}$ given in (42), the cognitive radio only needs to know the received SNR of the primary transmission at the primary base-station: $|p|^{2} P_{p} / N_{p}$. If the primary system uses a good (capacity-achieving) AWGN channel code and the cognitive radio knows this, the cognitive radio can easily compute an estimate of this received SNR since it knows the rate at which the primary user is communicating, $R_{p}$ : this estimate is simply given by $e^{R_{p}}-1$. Hence, the assumption that the cognitive radio has knowledge of the received SNR of the primary transmission is implied by the assumption that the cognitive radio knows the (capacity achieving) codebook of the primary radio.

\subsubsection{Achieving the fast-fading capacity at low-SNR}

The optimal cognitive communication scheme - both in the full-channel-state-information scenario, given by (32), and in the "fast-fading" scenario, given by (36) - requires that the cognitive radio's signal is perfectly time-synchronized with the primary radio's signal when it arrives at the primary base-station: i.e.,

$$
Y_{p}[m]=p X_{p}[m]+f X_{p}[m]+f \sqrt{\alpha \frac{P_{p}}{P_{c}}} \hat{X}_{c}[m]+Z_{p}[m] .
$$

In practice however, the delays from the two radios to the primary base-station could be different ${ }^{15}$, resulting in a received signal given by

$$
Y_{p}[m]=p X_{p}\left[m-l_{p}\right]+f \sqrt{\alpha \frac{P_{c}}{P_{p}}} X_{p}\left[m-l_{c}\right]+Z_{\text {total }}[m]
$$

where $Z_{\text {total }}[m]=f \hat{X}_{c}\left[m-l_{c}\right]+Z_{p}[m]$ is the aggregate noise. Hence, to achieve perfect synchronization, the cognitive radio would have to advance its signal by $l_{c}-l_{p}$. Given that the performance of the primary system is at stake, the estimates for $l_{c}$ and $l_{p}$ would need to be as accurate as possible.

However, one can circumvent the sensitive and risky task of time-synchronization by exploiting the built-in multipath resilience of the primary receiver. Observe that equation (44) essentially describes a time-invariant two-tap multipath (or ISI) channel for the primary transmission. The primary system is naturally designed to tolerate a

\footnotetext{
${ }^{15}$ Even though the two radios are relatively close, their signal paths could easily fall into different taps in a very high bandwidth system.
} 
certain amount of delay spread ${ }^{16}$ in the wireless channel. Transmit-receive architectures such as OFDM, or receivers such as the RAKE, in the case of direct-sequence spreadspectrum, are two very common methods used to combat multipath fading in a wireless channel. Both of these schemes would yield ${ }^{17}$ a power gain of $|p|^{2} P_{p}+|f|^{2} \alpha P_{c}$ at the primary base-station (see, for instance, Chapter 3 of [21], and references therein), thus achieving the rate (39) for the primary radio, at low SNR.

The received signal at the secondary base-station, on the other hand, is given by

$$
Y_{p}[m]=c \hat{X}_{c}\left[m-\widetilde{l}_{c}\right]+\left(c \sqrt{\alpha \frac{P_{c}}{P_{p}}} X_{p}\left[m-\widetilde{l}_{c}\right]+g X_{p}\left[m-\widetilde{l}_{p}\right]\right)+Z_{s}[m] .
$$

Hence, to achieve the "fast-fading" capacity promised in (43), $\hat{X}_{c}^{n}$ must be formed by Costa precoding for the interference term in parenthesis in (45). In order to do this, the cognitive radio must know the delays of the two radios with respect to the secondary base-station: $\widetilde{l}_{c}$ and $\widetilde{l}_{c}$. In a stationary environment, these values can be accurately tracked by the secondary base-station and fed-back to the cognitive radio. Hence, the capacity of the fast-fading cognitive radio channel, $R_{c}=\log \left(1+|c|^{2}\left(1-\alpha_{*}\right) P_{c} / N_{s}\right)$, is achieved.

\subsubsection{Discreetly entering the primary spectrum}

Though the expression for $\alpha_{*}$ in (42) does not depend on $|f|$, we can see that (41) can approximately be satisfied even with $\alpha=0$ when $|f|^{2}$ is very small. In other words, when $|f|$ is small, $\log \left(1+|c|^{2}\left(1-\alpha_{*}\right) P_{c} / N_{s}\right)$ may be a very conservative estimate for the maximum achievable rate for the cognitive radio.

Since the cognitive radio has no information about $|f|$, a natural way for the cognitive radio to enter the spectrum of the primary system would be by first setting $\alpha=0$ (transmitting purely its own information) and slowly ramping up its transmit power, from 0 to the maximum $P_{c}$, while listening for a NAK signal from the primary base-station. If a NAK is detected, the cognitive radio should then slightly decrease its transmit power and continue monitoring the ARQ channel to ensure that its new transmit power level is acceptable ${ }^{18}$. This new power level, call it $P(k)$, should then be compared with the quantity $\left(1-\alpha_{*}\right) P_{c}$ : if $P(k)$ is larger, it is kept as the new transmit power of the cognitive radio (with $\alpha=0$ ). However, if $\left(1-\alpha_{*}\right) P_{c}>P(k)$, the cognitive radio could achieve a

\footnotetext{
${ }^{16}$ In other words, the channel from the primary radio to its receiver is frequency selective with multiple taps. For simplicity of notation, we are only displaying the tap corresponding to the most dominant path.

${ }^{17}$ The total delay spread between the primary and cognitive radio transmission must be less than the maximum tolerable delay spread in the primary system: $\left(l_{c}-l_{p}\right) / W<T_{d} \approx 10 \mu \mathrm{s}$.

${ }^{18}$ The NAK signal may be caused by interference from sources other than the cognitive radio. If no more NAKs are transmitted after the cognitive radio reduces its power, and if the power of the primary radio returns to its nominal value, the cognitive radio can be sure that it indeed was the culprit.
} 
higher rate by using its maximum transmit power while still ensuring that the primary rate is unaffected: i.e., it should ramp up its power from $P(k)$ to $P_{c}$ while increasing $\alpha$ from 0 to $\alpha_{*}$.

\subsection{Obtaining the channel-state information}

In order to implement the optimal communication scheme of Costa coding and beamforming (32), the cognitive radio must obtain estimates of $p$ and $f$ from the primary base-station. If the primary radio is, by default, able to obtain estimates of its channel to the primary base-station (both magnitude and phase) via feed-back from the primary base-station, the cognitive radio could also potentially decode and use this information. ${ }^{19}$ Under this assumption, we present the following simple algorithm that the cognitive radio can use to acquire the estimates of $p$ and $f$.

1. At first, the cognitive user is silent and the primary base-station broadcasts the current estimate of $p$, call it $\hat{p}$, along with the primary user's ID, on the uplink control channel to which the cognitive radio is tuned. The primary base-station is assumed to be able to track $p$ by either using a pilot signal or in a decision-directed fashion. Thus, the cognitive radio can obtain $\hat{p}$.

2. Upon entering the system and decoding the message of the primary user in its vicinity, the cognitive radio simply performs amplify-and-forward relaying of the primary codeword:

$$
X_{c}^{n}=\sqrt{\alpha \frac{P_{c}}{P_{p}}} X_{p}^{n},
$$

where $\alpha \in[0,1]$.

3. The primary base-station receives

$$
\left(p+f \sqrt{\alpha \frac{P_{c}}{P_{p}}}\right) X_{p}^{n}+Z_{p}^{n},
$$

and computes an estimate, $\hat{h}$, of the overall channel gain $\left(p+f \sqrt{\alpha \frac{P_{c}}{P_{p}}}\right)$ as it decodes $m_{p}$.

4. The quantized version of $\hat{h}$ is then broadcast on the control channel, in the usual way, along with the given primary user's ID.

5. The cognitive radio picks up this information from the control channel and then computes $\hat{h}-\hat{p}$.

\footnotetext{
${ }^{19}$ The cognitive radio would have to be able to decode the dedicated control channel bits of the particular primary user in question.
} 
6. The quantity $\hat{h}-\hat{p}$ is an estimate for $f \sqrt{\alpha P_{c} / P_{p}}$ which is then multiplied by $\sqrt{\alpha P_{p} / P_{c}}$, to obtain an estimate for $f$.

Observe that a large $\alpha$ would cause a greater probability of a deep fade for the primary user since the quantity $\left|p+f \sqrt{\alpha P_{c} / P_{p}}\right|$, in step 3 above, would be more likely to be much smaller than $|p|$. In this case the primary system would momentarily not be able to support the requested rate of $\log \left(1+|p|^{2} P_{p} / N_{p}\right)$ and a NAK would be generated by the primary base-station. The conservative solution is for the cognitive radio to use a small value of $\alpha$ and soft-combine several consecutive estimates of $f$, while simultaneously listening for the primary base-station NAK signals. As soon as the first NAK is detected, the $\alpha$ parameter should either be reduced or set to 0 . In the case of a time-varying channel, the sequence of steps 1 through 6 would need to be performed periodically.

Finally, note that the capacity of the cognitive radio channel $R_{c}^{*}$ in Theorem 3.1 can only be achieved under the assumption of perfect channel state-estimation and feedback. In practice, the estimation and quantization errors will reduce the achievable rate and their effect can be modeled as additional independent Gaussian noise.

\section{A Proof of the converse part of Theorem 3.2}

First we observe that the rate-region specified in Theorem 3.2 is a convex set in Proposition D.1. We will use the following standard result from convex analysis (see, for instance, [17]) in the proof of the converse.

Proposition A.1 A point $\mathbf{R}^{*}=\left(R_{p}^{*}, R_{c}^{*}\right)$ is on the boundary of the a capacity region if and only if there exists a $\mu \geq 0$ such that the linear functional $\mu R_{p}+R_{c}$ achieves its maximum, over all $\left(R_{p}, R_{c}\right)$ in the region, at $\mathbf{R}^{*}$.

\section{A.1 The $\mu \leq 1$ case}

For convenience, we will consider a channel whose output at the primary receiver is normalized by $a$, i.e., a channel whose input-output single-letter equations are given by

$$
\begin{aligned}
Y_{p}^{n} & =\frac{1}{a} X_{p}^{n}+X_{c}^{n}+\frac{1}{a} Z_{p}^{n}, \\
Y_{s}^{n} & =b X_{p}^{n}+X_{c}^{n}+Z_{s}^{n} .
\end{aligned}
$$

Note that the capacity region of this channel is the same as that of the original channel (4), (5) since normalization is an invertible transformation. 
Suppose that a rate pair $\left(R_{p}, R_{c}\right)$ is achievable, in the sense of Definition 3.2 , for the $(1, a, b, 1)$-IC-DMS. Assuming that the messages $\left(m_{p}, m_{c}\right)$ are chosen uniformly and independently, we have, by Fano's inequality, $H\left(m_{p} \mid Y_{p}^{n}\right) \leq n \epsilon_{p, n}$ and $H\left(m_{c} \mid Y_{s}^{n}\right) \leq n \epsilon_{s, n}$, where $\epsilon_{p, n} \rightarrow 0$ and $\epsilon_{s, n} \rightarrow 0$ as $\widetilde{P}_{e, p}^{(n)} \rightarrow 0, P_{e, s}^{(n)} \rightarrow 0$, respectively. We start with the following bound on $n R_{p}$ :

$$
\begin{aligned}
n R_{p} & \stackrel{(a)}{=} H\left(m_{p}\right) \\
& =I\left(m_{p} ; Y_{p}^{n}\right)+H\left(m_{p} \mid Y_{p}^{n}\right) \\
& \stackrel{(b)}{\leq} I\left(m_{p} ; Y_{p}^{n}\right)+n \epsilon_{p, n} \\
& =h\left(Y_{p}^{n}\right)-h\left(Y_{p}^{n} \mid m_{p}\right)+n \epsilon_{p, n}
\end{aligned}
$$

where $(a)$ follows since $m_{p}$ and $m_{c}$ are uniformly distributed on $\left\{1,2, \ldots, 2^{n R_{p}}\right\}$ and $\left\{1,2, \ldots, 2^{n R_{p}}\right\}$ respectively, $(b)$ follows from Fano's inequality. Also, we have that,

$$
\begin{aligned}
n R_{c} & =H\left(m_{c}\right) \\
& =H\left(m_{c}\right)+H\left(m_{c} \mid Y_{s}^{n}, m_{p}\right)-H\left(m_{c} \mid Y_{s}^{n}, m_{p}\right) \\
& =I\left(m_{c} ; Y_{s}^{n} \mid m_{p}\right)+H\left(m_{c} \mid Y_{s}^{n}, m_{p}\right) \\
& \stackrel{(a)}{\leq} I\left(m_{c} ; Y_{s}^{n} \mid m_{p}\right)+n \epsilon_{s, n} \\
& =h\left(Y_{s}^{n} \mid m_{p}\right)-h\left(Y_{s}^{n} \mid m_{p}, m_{c}\right)+n \epsilon_{s, n} \\
& \stackrel{(b)}{\leq} h\left(Y_{s}^{n} \mid m_{p}\right)-h\left(Y_{s}^{n} \mid m_{p}, m_{c}, X_{p}^{n}, X_{c}^{n}\right)+n \epsilon_{s, n} \\
& \stackrel{(c)}{=} h\left(Y_{s}^{n} \mid m_{p}\right)-h\left(Z_{s}^{n}\right)+n \epsilon_{s, n},
\end{aligned}
$$

where $(a)$ follows from Fano's inequality and the fact that conditioning does not increase entropy, (b) follows from the fact that conditioning does not increase entropy, and $(c)$ follows from the the fact that $Z_{s}^{n}$ is independent of $\left(m_{p}, m_{c}\right)$ and hence also of $\left(X_{p}^{n}, X_{c}^{n}\right)$.

Let $\widetilde{Z}^{n}$ be a zero mean Gaussian random vector, independent of $\left(X_{p}^{n}, X_{c}^{n}, Z_{p}^{n}, Z_{s}^{n}\right)$ and with covariance matrix $\left(\frac{1}{a^{2}}-1\right) \mathbf{I}_{n}$. Then, we can write

$$
\begin{aligned}
h\left(\hat{Y}_{p}^{n} \mid m_{p}\right) & \stackrel{(a)}{=} h\left(\hat{Y}_{p}^{n} \mid m_{p}, X_{p}^{n}\right) \\
& \stackrel{(b)}{=} h\left(\hat{Y}_{p}^{n}-\frac{1}{a} X_{p}^{n} \mid m_{p}, X_{p}^{n}\right) \\
& =h\left(X_{c}^{n}+\frac{1}{a} Z_{p}^{n} \mid m_{p}, X_{p}^{n}\right) \\
& \stackrel{(c)}{=} h\left(X_{c}^{n}+Z_{s}^{n}+\widetilde{Z}^{n} \mid m_{p}, X_{p}^{n}\right) \\
& \stackrel{(d)}{=} h\left(X_{c}^{n}+Z_{s}^{n}+\widetilde{Z}^{n} \mid m_{p}\right) \\
& \stackrel{(e)}{=} h\left(\widetilde{Y}^{n}+\widetilde{Z}^{n} \mid m_{p}\right),
\end{aligned}
$$


where $(a)$ and $(d)$ hold since $X_{p}^{n}$ is the output of a deterministic function ${ }^{20}$ of $m_{p}$, (b) holds because translation does not affect entropy, $(c)$ follows from the fact that Gaussian distributions are infinitely divisible and from the definition of $\widetilde{Z}^{n}$ and $(e)$ follows from the definition $\widetilde{Y}^{n} \stackrel{\text { def }}{=} X_{c}^{n}+Z_{s}^{n}$. By similar reasoning, we can write

$$
h\left(Y_{s}^{n} \mid m_{p}\right)=h\left(\tilde{Y}^{n} \mid m_{p}\right) .
$$

Combining the bounds in (50) and (51), we get

$$
\begin{aligned}
n\left(\mu R_{p}+R_{c}\right) & \leq \mu\left(h\left(Y_{p}^{n}\right)-h\left(Y_{p}^{n} \mid m_{p}\right)\right)+h\left(Y_{s}^{n} \mid m_{p}\right)-h\left(Z_{s}^{n}\right)+\mu n \epsilon_{p, n}+n \epsilon_{s, n} \\
& \stackrel{(a)}{=} \mu h\left(Y_{p}^{n}\right)+h\left(Y_{s}^{n} \mid m_{p}\right)-\mu h\left(Y_{p}^{n} \mid m_{p}\right)-\frac{n}{2} \log (2 \pi e)+\mu n \epsilon_{p, n}+n \epsilon_{s, n} \\
& \stackrel{(b)}{=} \mu h\left(Y_{p}^{n}\right)+h\left(\widetilde{Y}^{n} \mid m_{p}\right)-\mu h\left(\widetilde{Y}^{n}+\widetilde{Z}^{n} \mid m_{p}\right)-\frac{n}{2} \log (2 \pi e)+\mu n \epsilon_{p, n}+n \epsilon_{s, n} \\
& \stackrel{(c)}{\leq} \mu h\left(Y_{p}^{n}\right)+h\left(\widetilde{Y}^{n} \mid m_{p}\right)-\frac{\mu n}{2} \log \left(e^{\frac{2}{n} h\left(\widetilde{Y}^{n} \mid m_{p}\right)}+e^{\frac{2}{n} h\left(\widetilde{Z}^{n}\right)}\right) \\
& -\frac{n}{2} \log (2 \pi e)+\mu n \epsilon_{p, n}+n \epsilon_{s, n},(54)
\end{aligned}
$$

where $(a)$ follows from the fact that $Z_{s}^{n} \sim \mathcal{N}\left(0, \mathbf{I}_{n}\right)$, (b) follows from equalities (52) and (53), $(c)$ follows from the conditional version of the Entropy Power Inequality (see Proposition I.1 of [22]).

Let $X_{1}^{j-1}$ denote the first $j-1$ components of the vector $X^{n}$ with the understanding that $X_{1}^{0}$ is defined to be some constant and let $X_{j}$ denote the $j$-th component. We can upper-bound $h\left(\widetilde{Y}^{n} \mid m_{p}\right)$ as follows:

$$
\begin{aligned}
& h\left(\tilde{Y}^{n} \mid m_{p}\right)=h\left(\tilde{Y}^{n} \mid m_{p}, X_{p}^{n}\right) \\
& \stackrel{(a)}{=} \sum_{j=1}^{n} h\left(\widetilde{Y}_{j} \mid m_{p}, \widetilde{Y}_{1}^{j-1}, X_{p, j}, X_{p, 1}^{j-1}, X_{p, j+1}^{n}\right) \\
& \stackrel{(b)}{\leq} \sum_{j=1}^{n} h\left(\tilde{Y}_{j} \mid X_{p, j}\right) \\
& \stackrel{(c)}{\leq} \sum_{j=1}^{n} \frac{1}{2} \log \left(2 \pi e\left(\mathbb{E}\left[\widetilde{Y}_{j}^{2}\right]-\frac{\mathbb{E}\left[\tilde{Y}_{j} X_{p, j}\right]^{2}}{\mathbb{E}\left[X_{p, j}^{2}\right]}\right)\right) \\
& \stackrel{(d)}{=} \sum_{j=1}^{n} \frac{1}{2} \log \left(2 \pi e\left(\left(1-\alpha_{j}\right) P_{c, j}+1\right)\right) \\
& \stackrel{(e)}{\leq} \frac{n}{2} \log \left(2 \pi e\left((1-\alpha) P_{c}+1\right)\right) \text {, }
\end{aligned}
$$

\footnotetext{
${ }^{20}$ In general, the encoder $E_{p}^{n}$ could be stochastic, i.e., depend on a random variable generated at the primary radio. In this case, inequalities (50) and (51) would need to include conditioning on $E_{p}^{n}$ in addition to $m_{p}$ (note that this can be done for free in (51) while it would yield a further upper bound in (50)). Since the steps in the proof would be the same, we assume that the encoders are deterministic to simplify the notation.
} 
where $(a)$ follows from the chain rule and $(b)$ follows from the fact that conditioning does not increase entropy, and $(c)$ follows from Lemma 1 of [20] (a more direct proof appears in Lemma 1 of $[19])$. Equality $(d)$ follows from the following argument: Since jointly Gaussian $X_{p, j}, \tilde{Y}_{p, j}$ achieve equality in (c) (by Lemma 1 of [20]), we can without loss of generality, let

$$
X_{c, j}=\hat{X}_{c, j}+\sqrt{\alpha_{j} \frac{P_{c, j}}{P_{p, j}}} X_{p, j}
$$

where $\hat{X}_{c, j} \sim \mathcal{N}\left(0,\left(1-\alpha_{j}\right) P_{c, j}\right)$ is independent of $X_{p, j}$ and

$$
P_{c, j} \stackrel{\text { def }}{=} \frac{1}{2^{n R_{c}}} \sum_{j=1}^{2^{n R_{c}}} X_{c, j}^{2}, \quad P_{p, j} \stackrel{\text { def }}{=} \frac{1}{2^{n R_{p}}} \sum_{j=1}^{2^{n R_{p}}} X_{p, j}^{2} .
$$

The parameter $\alpha_{j} \in[0,1]$ is chosen so that the resulting covariance $K_{X_{p, j}, X_{c, j}, Y_{s, j}, Y_{p, j}}$ is the same as that induced by the code. Inequality labeled with $(e)$ follows from Jensen's inequality, by choosing $\alpha \in[0,1]$ such that

$$
\alpha P_{c}=\frac{1}{n} \sum_{j=1}^{n} \alpha_{j} P_{c, j}
$$

and from the fact that the power constraint $\left\|X_{c}^{n}\right\|^{2} \leq n P_{c}$ implies that $\frac{1}{n} \sum_{j=1}^{n} P_{c, j}=P_{c}$.

Similarly, we can upper bound $h\left(Y_{p}\right)$ as follows:

$$
\begin{aligned}
h\left(Y_{p}^{n}\right) & \stackrel{(a)}{=} \sum_{j=1}^{n} h\left(Y_{p, j} \mid Y_{p, 1}^{j-1}\right) \\
& \stackrel{(b)}{\leq} \sum_{j=1}^{n} h\left(Y_{p, j}\right) \\
& \stackrel{(c)}{\leq} \sum_{j=1}^{n} \frac{1}{2} \log \left(2 \pi e \mathbb{E}\left[Y_{p, j}^{2}\right]\right) \\
& \stackrel{(d)}{=} \sum_{j=1}^{n} \frac{1}{2} \log \left(\frac{2 \pi e}{a^{2}}\left(P_{p, j}+2 a \sqrt{\alpha_{j} P_{p, j} P_{c, j}}+a^{2} P_{c, j}+1\right)\right) \\
& \stackrel{(e)}{\leq} \frac{n}{2} \log \left(\frac{2 \pi e}{a^{2}}\left(\left(\sqrt{P_{p}}+a \sqrt{\alpha P_{c}}\right)^{2}+a^{2}(1-\alpha) P_{c}+1\right)\right),
\end{aligned}
$$

where $(a)$ follows from the chain rule and $(b)$ follows from the fact that conditioning does not increase entropy, $(c)$ holds since the Gaussian distribution maximizes the differential entropy for a fixed variance, $(d)$ follows from the same argument as in (55) and $(e)$ comes from Jensen's inequality applied to the $\log (\cdot)$ and the $\sqrt{ } \cdot$ functions. 
Let $f(x) \stackrel{\text { def }}{=} x-\frac{\mu n}{2} \log \left(e^{\frac{2}{n} x}+e^{\frac{2}{n} h\left(\widetilde{Z}^{n}\right)}\right)$ over $x \in \mathbb{R}$. Then, we can express the bound on our linear functional in (54) as

$$
n\left(\mu R_{p}+R_{c}\right) \leq \mu h\left(Y_{p}^{n}\right)+f\left(h\left(\tilde{Y}^{n} \mid m_{p}\right)\right)-\frac{n}{2} \log (2 \pi e)+\mu n \epsilon_{p, n}+n \epsilon_{s, n} .
$$

Observe that as long as $\mu \leq 1, f(x)$ is increasing. Hence we can obtain a further upper bound by substituting inequalities (56) and (60) into (61):

$$
\begin{aligned}
n\left(\mu R_{p}+R_{c}\right) \leq & \mu \frac{n}{2} \log \left(\frac{2 \pi e}{a^{2}}\left(\left(\sqrt{P_{p}}+a \sqrt{\alpha P_{c}}\right)^{2}+a^{2}(1-\alpha) P_{c}+1\right)\right) \\
& +f\left(\frac{n}{2} \log \left(2 \pi e\left((1-\alpha) P_{c}+1\right)\right)\right)-\frac{n}{2} \log (2 \pi e)+\mu n \epsilon_{p, n}+n \epsilon_{s, n}(63) \\
\stackrel{(a)}{=} & \mu \frac{n}{2} \log \left(\frac{2 \pi e}{a^{2}}\left(\left(\sqrt{P_{p}}+a \sqrt{\alpha P_{c}}\right)^{2}+a^{2}(1-\alpha) P_{c}+1\right)\right) \\
& +\frac{n}{2} \log \left(2 \pi e\left((1-\alpha) P_{c}+1\right)\right)-\mu \frac{n}{2} \log \left(2 \pi e\left((1-\alpha) P_{c}+\frac{1}{a^{2}}\right)\right) \\
& -\frac{n}{2} \log (2 \pi e)+\mu n \epsilon_{p, n}+n \epsilon_{s, n},
\end{aligned}
$$

where $(a)$ follows from the fact that

$$
\begin{aligned}
f(x) & =x-\frac{\mu n}{2} \log \left(e^{\frac{2}{n} x}+e^{\frac{2}{n} h\left(\widetilde{Z}^{n}\right)}\right) \\
& =x-\frac{\mu n}{2} \log \left(e^{\frac{2}{n} x}+2 \pi e\left(\frac{1}{a^{2}}-1\right)\right),
\end{aligned}
$$

which holds since $\widetilde{Z}^{n}$ is zero mean Gaussian with covariance $\left(\frac{1}{a^{2}}-1\right) \mathbf{I}$.

Grouping together the $\mu$-terms, dividing by $n$ and letting $n \rightarrow \infty$, we get that

$$
\mu R_{p}+R_{c} \leq \frac{\mu}{2} \log \left(1+\frac{\left(\sqrt{P_{p}}+a \sqrt{\alpha P_{c}}\right)^{2}}{1+a^{2}(1-\alpha) P_{c}}\right)+\frac{1}{2} \log \left(1+(1-\alpha) P_{c}\right) .
$$

Let $\alpha_{\mu}$ denote the maximizing $\alpha \in[0,1]$ for a given $\mu \leq 1$ in the above expression. Then, we can write

$$
\mu R_{p}+R_{c} \leq \frac{\mu}{2} \log \left(1+\frac{\left(\sqrt{P_{p}}+a \sqrt{\alpha_{\mu} P_{c}}\right)^{2}}{1+a^{2}\left(1-\alpha_{\mu}\right) P_{c}}\right)+\frac{1}{2} \log \left(1+\left(1-\alpha_{\mu}\right) P_{c}\right)
$$

Hence we have established the converse of the theorem for $\mu \leq 1$.

\section{A.2 The $\mu \geq 1$ case}

An outer bound to the boundary of the capacity region for the $\mu \geq 1$ case can be found in [11]. The original version of our paper contained this bound as a converse, but the bound was found to be not tight in general. 


\section{B Proof of Corollary 4.1}

The proof of this Corollary follows from Theorem 3.2 and Lemma D.1. In particular, we observe that the converse to Theorem 3.2 for $\mu \geq 1$ (see Section A.2) holds for any $a>0$ and $b \in \mathbb{R}$. However, from Lemma D.1 we see that the choice $\alpha=1$ in (25) and (26) is optimal for any $a \geq 1$, as long as $\mu \geq 1$. Hence the corollary is proved.

Remark: This result implies that, for any $a \geq 1, b \in \mathbb{R}$ and $\mu \geq 1$, the linear functional $\mu R_{p}+R_{c}$ is maximized at $\left(R_{p}, R_{c}\right)=\left(C_{\text {sum }}(a), 0\right)$. Hence, for $a \geq 1$, the entire capacity region is parametrized by $\mu \leq 1$, for any $b \in \mathbb{R}$.

\section{Proof of the converse part of Theorem 4.2}

Let "genie B" disclose $m_{c}$ to the primary transmitter, thus getting a $2 \times 1$ MIMO BC channel with per-antenna power constraints. The input-output relationship for this channel can be written as

$$
\begin{aligned}
& Y_{p}=\mathbf{h}_{p}^{T} \mathbf{X}+Z_{p}, \\
& Y_{s}=\mathbf{h}_{s}^{T} \mathbf{X}+Z_{s},
\end{aligned}
$$

where $\mathbf{h}_{p}=\left[\begin{array}{ll}1 & a\end{array}\right]^{T}$ and $\mathbf{h}_{s}=\left[\begin{array}{ll}b & 1\end{array}\right]^{T}$. We choose $\mu \leq 1$ in the linear functional $\mu R_{p}+R_{c}$ and recall that the optimal transmission vector $\mathbf{X}$ is Gaussian and given by

$$
\mathbf{X}=X_{p 1} \mathbf{u}_{p 1}+X_{p 2} \mathbf{u}_{p 2}+X_{c 1} \mathbf{u}_{c 1}+X_{c 2} \mathbf{u}_{c 2},
$$

where $\mathbf{u}_{p 1}, \mathbf{u}_{p 2} \in \mathbb{R}^{2}$ and $\mathbf{u}_{c 1}, \mathbf{u}_{c 2} \in \mathbb{R}^{2}$ are the so-called signature vectors and symbols $X_{p 1}, X_{p 2}$ and $X_{c 1}, X_{c 2}$ are i.i.d. $\mathcal{N}(0,1)$.

In order to emulate the per-user individual power constraints of the IC-DMS, we impose the per-antenna constraints $\left(\mathbb{E}\left[\mathbf{X X}^{T}\right]\right)_{11} \leq P_{p}$ and $\left(\mathbb{E}\left[\mathbf{X X}^{T}\right]\right)_{22} \leq P_{c}$ on the achievable strategies in MIMO BC channel. We let

$$
\begin{array}{ll}
\boldsymbol{\Sigma}_{p} & \stackrel{\text { def }}{=} \\
\boldsymbol{\Sigma}_{c} & \mathbf{u}_{p 1} \mathbf{u}_{p 1}^{T}+\mathbf{u}_{p 2} \mathbf{u}_{p 2}^{T}, \\
= & \mathbf{u}_{c 1} \mathbf{u}_{c 1}^{T}+\mathbf{u}_{c 2} \mathbf{u}_{c 2}^{T},
\end{array}
$$

so that, by the independence of $X_{p 1}, X_{p 2}, X_{c 1}$ and $X_{c 2}$, the constraint can be expressed as $\left(\Sigma_{p}+\Sigma_{c}\right)_{11} \leq P_{p}$ and $\left(\Sigma_{p}+\Sigma_{c}\right)_{22} \leq P_{c}$.

From [22], we know that the optimal encoding strategy is to generate $X_{p}$ by Costa 
pre-coding ${ }^{21}$ for $\mathbf{h}_{p}^{T}\left(X_{c 1} \mathbf{u}_{c 1}+X_{c 2} \mathbf{u}_{c 2}\right)$. The rates achievable with such a scheme are:

$$
\begin{aligned}
& R_{p}=\hat{R}_{p}\left(\boldsymbol{\Sigma}_{p}^{*}, \boldsymbol{\Sigma}_{c}^{*}\right) \stackrel{\text { def }}{=} \frac{1}{2} \log \left(1+\mathbf{h}_{p}^{T} \boldsymbol{\Sigma}_{p}^{*} \mathbf{h}_{p}\right), \\
& R_{c}=\hat{R}_{c}\left(\boldsymbol{\Sigma}_{p}^{*}, \boldsymbol{\Sigma}_{c}^{*}\right) \stackrel{\text { def }}{=} \frac{1}{2} \log \left(1+\frac{\mathbf{h}_{s}^{T} \boldsymbol{\Sigma}_{c}^{*} \mathbf{h}_{s}}{1+\mathbf{h}_{s}^{T} \boldsymbol{\Sigma}_{p}^{*} \mathbf{h}_{s}}\right),
\end{aligned}
$$

where $\boldsymbol{\Sigma}_{p}^{*}$ and $\boldsymbol{\Sigma}_{c}^{*}$ are the solutions of

$$
\arg \max _{\left(\boldsymbol{\Sigma}_{p}, \boldsymbol{\Sigma}_{c}\right) \in \mathcal{S}\left(P_{p}, P_{c}\right)} \mu R_{p}\left(\boldsymbol{\Sigma}_{p}, \boldsymbol{\Sigma}_{c}\right)+R_{c}\left(\boldsymbol{\Sigma}_{p}, \boldsymbol{\Sigma}_{c}\right)
$$

where $\mu \leq 1$ and $\mathcal{S}\left(P_{p}, P_{c}\right) \stackrel{\text { def }}{=}\left\{\boldsymbol{\Sigma}_{p} \succeq 0, \boldsymbol{\Sigma}_{c} \succeq 0:\left(\boldsymbol{\Sigma}_{p}+\boldsymbol{\Sigma}_{c}\right)_{11} \leq P_{p},\left(\boldsymbol{\Sigma}_{p}+\boldsymbol{\Sigma}_{c}\right)_{22} \leq P_{c}\right\}$.

Since the per-antenna power constraints must be met with equality, ${ }^{22}$ we can, without loss of generality, write

$$
\begin{aligned}
& \boldsymbol{\Sigma}_{p}=\left[\begin{array}{cc}
\beta P_{p} & k_{p} \\
k_{p} & \alpha P_{c}
\end{array}\right], \quad \text { where } k_{p} \in\left[-\sqrt{\alpha \beta P_{p} P_{c}}, \sqrt{\alpha \beta P_{p} P_{c}}\right] \\
& \boldsymbol{\Sigma}_{c}=\left[\begin{array}{cc}
(1-\beta) P_{p} & k_{c} \\
k_{c} & (1-\alpha) P_{c}
\end{array}\right], \quad \text { where } k_{c} \in\left[-\sqrt{\bar{\alpha} \bar{\beta} P_{p} P_{c}}, \sqrt{\bar{\alpha} \bar{\beta} P_{p} P_{c}}\right]
\end{aligned}
$$

and $\beta \in[0,1], \alpha \in[0,1]$ and $\bar{\alpha} \stackrel{\text { def }}{=} 1-\alpha, \bar{\beta} \stackrel{\text { def }}{=} 1-\beta$.

Substituting the covariance matrices (79) and (80) into (76) and (77), we get

$$
\begin{array}{ll}
\hat{R}_{p}\left(\boldsymbol{\Sigma}_{p}, \boldsymbol{\Sigma}_{c}\right)=\hat{R}_{p}\left(\beta, \alpha, k_{p}, a, b\right) & \stackrel{\text { def }}{=} \frac{1}{2} \log \left(1+\beta P_{p}+2 a k_{p}+\alpha a^{2} P_{c}\right) \\
\hat{R}_{c}\left(\boldsymbol{\Sigma}_{p}, \boldsymbol{\Sigma}_{c}\right) & =\hat{R}_{c}\left(\beta, \alpha, k_{p}, a, b\right) \stackrel{\text { def }}{=} \frac{1}{2} \log \left(1+\frac{b^{2}(1-\beta) P_{p}+2 k_{c} b+(1-\alpha) P_{c}}{1+b^{2} \beta P_{p}+2 k_{p} b+\alpha P_{c}}\right)
\end{array}
$$

The expression in (82) is maximized by choosing $k_{c}=\sqrt{(1-\beta)(1-\alpha) P_{p} P_{c}}$, i.e., making $\Sigma_{c}$ unit rank. If $b=0$ it is clear that $\beta=1$ and $k_{p}=\sqrt{\alpha P_{p} P_{c}}$ maximizes the linear functional $\mu \hat{R}_{p}\left(\beta, \alpha, k_{p}, a, b\right)+\hat{R}_{c}\left(\beta, \alpha, k_{p}, a, b\right)$. In general, we would like to find the set of all values of $b$ for which $\beta=1$ and $k_{p}=\sqrt{\alpha P_{p} P_{c}}$ are optimal. For such values of $b$, we then have

$$
\begin{aligned}
& \hat{R}_{p}\left(\boldsymbol{\Sigma}_{p}, \boldsymbol{\Sigma}_{c}\right)=\frac{1}{2} \log \left(1+\left(\sqrt{P_{p}}+a \sqrt{\alpha P_{c}}\right)^{2}\right), \\
& \hat{R}_{c}\left(\boldsymbol{\Sigma}_{p}, \boldsymbol{\Sigma}_{c}\right)=\frac{1}{2} \log \left(1+\frac{(1-\alpha) P_{c}}{1+\left(b \sqrt{P_{p}}+\sqrt{\alpha P_{c}}\right)^{2}}\right),
\end{aligned}
$$

\footnotetext{
${ }^{21}$ Costa's scheme is a block-coding scheme and, strictly speaking, encoding is performed on the vector $\left(X_{c 1}^{n}, X_{c 2}^{n}\right)$ given $X_{p 1}^{n}$ and $X_{p 2}^{n}$.

${ }^{22}$ If, instead, antenna 1 uses only $P_{p}-\eta$ power, we can add another antenna with power $\eta$ whose signal the receivers can first decode and then subtract off thus boosting at least one of the rates. The same applies to antenna 2.
} 
which exactly match the achievable rates given in Lemma 4.1. To this end, let $B(\mu, a)$ denote the set of all $b>0$ such that the function

$$
\max _{0 \leq \alpha \leq 1} \mu \hat{R}_{p}\left(\beta, \alpha, k_{p}, a, b\right)+\hat{R}_{c}\left(\beta, \alpha, k_{p}, a, b\right)
$$

is maximized, over all $\beta \in[0,1]$ and $k_{p} \in\left[-\sqrt{\beta \alpha P_{p} P_{c}}, \sqrt{\beta \alpha P_{p} P_{c}}\right]$, by choosing $\beta=1$ and $k_{p}=\sqrt{\alpha P_{p} P_{c}}$. We let $b_{\max }(\mu, a) \stackrel{\text { def }}{=} \max _{b \in B(\mu, a)}$ to obtain the statement of the theorem. Appealing to the remark in the proof of Corollary 4.1 (see Appendix B), we observe that the boundary of the capacity region in this very-high-interference-gain regime is completely parametrized by $\mu \leq 1$. Hence, we have proved the theorem.

\section{Supporting results}

Proposition D.1 The rate region specified in Theorem 3.2 is a convex set.

Proof: A point $\mathbf{R}=\left(R_{p}, R_{c}\right)$ is in the rate region specified in Theorem 3.2 if and only if there exists $\alpha \in[0,1]$ such that

$$
\begin{aligned}
R_{c} & \leq \frac{1}{2} \log \left(1+(1-\alpha) P_{c}\right) \\
R_{p}+R_{c} & \leq \frac{1}{2} \log \left(1+a^{2} P_{c}+P_{p}+2 a \sqrt{\alpha P_{p} P_{c}}\right)+\frac{1}{2} \log \left(\frac{1+(1-\alpha) P_{c}}{1+a^{2}(1-\alpha) P_{c}}\right)
\end{aligned}
$$

Suppose that there exist two points $\mathbf{R}^{(1)}=\left(R_{p}^{(1)}, R_{c}^{(1)}\right)$ and $\mathbf{R}^{(2)}=\left(R_{p}^{(2)}, R_{c}^{(2)}\right)$ that are on the boundary of this region. Let $\alpha^{(1)} \in[0,1]$ and $\alpha^{(2)} \in[0,1]$ be their corresponding parameters in (86) and (87) for which the inequalities become tight. Then for any $\lambda \in[0,1]$, we have that

$$
\begin{aligned}
\lambda R_{c}^{(1)}+(1-\lambda) R_{c}^{(2)} & \leq \frac{\lambda}{2} \log \left(1+\left(1-\alpha^{(1)}\right) P_{c}\right)+\frac{1-\lambda}{2} \log \left(1+\left(1-\alpha^{(2)}\right) P_{c}\right) \\
& \leq \frac{1}{2} \log \left(1+\left(1-\alpha^{*}\right) P_{c}\right)
\end{aligned}
$$

where $\alpha^{*} \stackrel{\text { def }}{=} \lambda \alpha^{(1)}+(1-\lambda) \alpha^{(2)}$ and the last inequality follows from Jensen's inequality. Similarly,

$$
\begin{aligned}
\lambda\left(R_{p}^{(1)}+R_{c}^{(1)}\right)+ & (1-\lambda)\left(R_{p}^{(2)}+R_{c}^{(2)}\right) \\
\leq \quad\left[\frac{\lambda}{2} \log (1+\right. & \left.\left.a^{2} P_{c}+P_{p}+2 a \sqrt{\alpha^{(1)} P_{p} P_{c}}\right)+\frac{1-\lambda}{2} \log \left(1+a^{2} P_{c}+P_{p}+2 a \sqrt{\alpha^{(2)} P_{p} P_{c}}\right)\right] \\
& +\left[\frac{\lambda}{2} \log \left(\frac{1+\left(1-\alpha^{(1)}\right) P_{c}}{1+a^{2}\left(1-\alpha^{(1)}\right) P_{c}}\right)+\frac{1-\lambda}{2} \log \left(\frac{1+\left(1-\alpha^{(2)}\right) P_{c}}{1+a^{2}\left(1-\alpha^{(2)}\right) P_{c}}\right)\right] . \quad(90)
\end{aligned}
$$


We can further upper bound (90) as follows:

$$
\begin{gathered}
\lambda\left(R_{p}^{(1)}+R_{c}^{(1)}\right)+(1-\lambda)\left(R_{p}^{(2)}+R_{c}^{(2)}\right) \\
\stackrel{(a)}{\leq} \frac{1}{2} \log \left(1+a^{2} P_{c}+P_{p}+2 a \sqrt{P_{p} P_{c}}\left(\lambda \sqrt{\alpha^{(1)}}+(1-\lambda) \sqrt{\alpha^{(2)}}\right)\right) \\
\quad+\frac{1}{2} \log \left(\frac{1+\left(1-\lambda \alpha^{(1)}-(1-\lambda) \alpha^{(2)}\right) P_{c}}{1+a^{2}\left(1-\lambda \alpha^{(1)}-(1-\lambda) \alpha^{(2)}\right) P_{c}}\right) \\
\stackrel{(\text { b) }}{\leq} \frac{1}{2} \log \left(1+a^{2} P_{c}+P_{p}+2 a \sqrt{P_{p} P_{c} \alpha^{*}}\right)+\frac{1}{2} \log \left(\frac{1+\left(1-\alpha^{*}\right) P_{c}}{1+a^{2}\left(1-\alpha^{*}\right) P_{c}}\right),
\end{gathered}
$$

where $(a)$ follows from Jensen's inequality applied to the concave function $\log \left(k_{1}+k_{2} x\right)$ (for constant $k_{1}, k_{2}>0$ ) and the concave function $\log \left(\frac{1+(1-x)}{1+k(1-x)}\right)$ (for constant $k \leq 1$ ). Inequality $(b)$ follows from Jensen's inequality applied to the square-root function. This shows that $\lambda \mathbf{R}^{(1)}+(1-\lambda) \mathbf{R}^{(2)}$ is in the region as well, hence the region is a convex set.

\section{Lemma D.1}

$$
\begin{aligned}
\max _{0 \leq \alpha \leq 1} \frac{\mu}{2} \log \left(1+\frac{\left(\sqrt{P_{p}}+a \sqrt{\alpha P_{c}}\right)^{2}}{1+a^{2}(1-\alpha) P_{c}}\right)+\frac{1}{2} \log \left(1+(1-\alpha) P_{c}\right) & \\
& =\frac{\mu}{2} \log \left(1+\left(\sqrt{P_{p}}+a \sqrt{P_{c}}\right)^{2}\right),
\end{aligned}
$$

for $a \geq 1$ and $\mu \geq 1$.

Proof: On the one hand, we have that

$$
\begin{aligned}
\max _{0 \leq \alpha \leq 1} \frac{\mu}{2} \log & \left(1+\frac{\left(\sqrt{P_{p}}+a \sqrt{\alpha P_{c}}\right)^{2}}{1+a^{2}(1-\alpha) P_{c}}\right)+\frac{1}{2} \log \left(1+(1-\alpha) P_{c}\right) \\
& =\max _{0 \leq \alpha \leq 1} \frac{1}{2} \log \left(\frac{\left(1+a^{2}(1-\alpha) P_{c}+\left(\sqrt{P_{p}}+a \sqrt{\alpha P_{c}}\right)^{2}\right)^{\mu}\left(1+(1-\alpha) P_{c}\right)}{\left(1+a^{2}(1-\alpha) P_{c}\right)^{\mu}}\right) \\
& \leq \max _{0 \leq \alpha \leq 1} \frac{1}{2} \log \left(\frac{\left(1+a^{2}(1-\alpha) P_{c}+\left(\sqrt{P_{p}}+a \sqrt{\alpha P_{c}}\right)^{2}\right)^{\mu}}{\left(1+a^{2}(1-\alpha) P_{c}\right)^{\mu-1}}\right) \\
& =\max _{0 \leq \alpha \leq 1} \frac{1}{2} \log \left(\frac{\left(1+a^{2} P_{c}+P_{p}+2 a \sqrt{\alpha P_{p} P_{c}}\right)^{\mu}}{\left(1+a^{2}(1-\alpha) P_{c}\right)^{\mu-1}}\right) \\
& =\frac{\mu}{2} \log \left(1+\left(\sqrt{P_{p}}+a \sqrt{P_{c}}\right)^{2}\right)
\end{aligned}
$$

On the other hand, the maximization problem in (91) can be lower bounded with $\frac{\mu}{2} \log \left(1+\left(\sqrt{P_{p}}+a \sqrt{P_{c}}\right)^{2}\right)$, by choosing $\alpha=1$. Hence the lemma is proved. 


\section{Acknowledgement}

The authors would like to thank Hesham El Gamal for pointing out an error error in the original version of our paper, in the proof of converse of the IC-DMS in the original formulation of Theorem (3.2) . The authors would also like to thank Patrick Mitran for his comments on the previous draft of this paper, Gerhard Kramer for his suggestion that led to Section 5.4.1, and the anonymous reviewers for their constructive comments and suggestions.

\section{References}

[1] A.B. Carleial, "Interference channels," IEEE Transactions on Information Theory, vol. 24, no. 1, pp. 60-70, Jan. 1978

[2] A. Cohen and A. Lapidoth, "The Gaussian watermarking game," IEEE Transactions on Information Theory, vol. 48, pp. 16391667, June 2002.

[3] M. H. M. Costa, "Writing on dirty paper," IEEE Transactions on Information Theory, vol. 29, no. 3, pp. 439-441, May 1983.

[4] H.M. Costa, "On the Gaussian interference channel," IEEE Transactions on Information Theory, vol. 31, no. 5, pp. 607-615, Sept. 1985.

[5] A. Dembo, T.M. Cover, and J.A. Thomas, "Information theoretic inequalities," IEEE Transactions on Information Theory, vol. 37, no. 6, pp. 1501-1518, Nov. 1991.

[6] N. Devroye, P. Mitran, and V. Tarokh, "Achievable Rates in Cognitive Channels," IEEE Transactions on Information Theory, vol. 52, no. 5, pp. 1813-1827, May 2006.

[7] Federal Communications Commission Spectrum Policy Task Force, "Report of the Spectrum Efficiency Working Group", FCC, Tech Report, Nov. 2002.

[8] Federal Communications Commision, Cognitive Radio Technologies Proceeding (CRTP), ET Docket No. 03-108, http://www.fcc.gov/oet/cognitiveradio/.

[9] IEEE Standards Association, "IEEE 802.16e Mobile WirelessMAN (R) Standard is Official" http://standards.ieee.org/announcements/pr_p80216.html, Dec. 2005.

[10] International Telecommunications Union draft, "Characteristics of the IEEE 802.16 systems in the $2500-2690 \mathrm{MHz}$ ", Dec. 2004, http://wirelessman.org/liaison/ docs/L80216-04_42r2.pdf

[11] A. Jovicic, P. Viswanath, "Cognitive Radio: An Information-Theoretic Perspective", http://arxiv.org/abs/cs/0604107 
[12] A. Lapidoth, "Nearest-neighbor decoding for additive non-Gaussian noise channels," IEEE Transactions on Information Theory, vol. 42, no. 5, pp. 1520-1529, Sept. 1996.

[13] I. Maric, R. D. Yates and G. Kramer, "The Strong Interference Channel With Unidirectional Cooperation," The Information Theory and Applications (ITA) Inaugural Workshop, La Jolla, CA, Feb. 2006.

[14] I. Maric, R. D. Yates and G. Kramer, "The Capacity Region of the Strong Interference Channel With Common Information," Asilomar Conference On Signals, Systems and Computers, Pacific Grove, CA, Nov. 2005.

[15] P. Mitran, H. Ochiai, V. Tarokh, "Space-Time Diversity Enhancements Using Collaborative Communications," in IEEE Transactions on Information Theory, vol. 51, no. 6, pp. 2041-2057, June 2005.

[16] J. M. Peha and S. Panichpapiboon, "Real-Time Secondary Markets for Spectrum," Telecommunications Policy, vol. 28, pp. 603-618, Aug.-Sep. 2004

[17] R. T. Rockafellar Convex Analysis, Princeton University Press, 1971.

[18] H. Sato, "The capacity of the Gaussian interference channel under strong interference," IEEE Transactions on Information Theory, vol. 27, no. 6, pp. 786-788, Nov. 1981.

[19] A. Sutivong, M. Chiang, T. M. Cover, Y.-H. Kim, "Channel capacity and state estimation for state-dependent Gaussian channels," IEEE Transactions on Information Theory, vol. 51, no. 4, pp. 1486-1495, April 2005.

[20] J. Thomas, "Feedback can at most double Gaussian multiple access channel capacity", IEEE Transactions on Information Theory, vol. 33, No.5, Sept. 1987.

[21] D. Tse and P. Viswanath, "Fundamentals of Wireless Communication", Cambridge University Press, 2005.

[22] H. Weingarten, Y. Steinberg, S. Shamai, "The Capacity Region of the Gaussian MIMO Broadcast Channel", submitted to IEEE Transactions on Information Theory, July 2004.

[23] W. Wu, S. Vishwanath, and A. Arapostathis, "On the Capacity of Gaussian Weak Interference Channels with Degraded Message Sets", Proc. of 40th Annual Conference on Information Sciences and Systems, March 2006.

[24] W. Wu, S. Vishwanath, A. Arapostathis, "Capacity of a Class of Cognitive Radio Channels: Interference Channels With Degraded Message Sets", IEEE Transactions on Information Theory, vol. 53, no. 11, pp. 4391-4399, Nov. 2007.

Aleksandar Jovičić received the B.S. degree in Electrical Engineering from the University of Wisconsin-Madison in 2001 and the M.S. and Ph.D. degrees in Electrical 
and Computer Engineering (ECE) from the University of Illinois at Urbana-Champaign in 2003 and 2007, respectively. He is currently a Staff Engineer at Qualcomm Flarion Technologies in Bridgewater, NJ. Aleksandar is the recipient of the Robert T. Chien Memorial Award (2007) for excellence in research from the department of ECE of the University of Illinois at Urbana-Champaign.

Pramod Viswanath (S98A00M03) received the Ph.D. degree in electrical engineering and computer science from the University of California at Berkeley in 2000. He is an Associate Professor in the Electrical and Computer Engineering (ECE) Department at the University of Illinois at Urbana-Champaign, Urbana, IL. He was a Member of Technical Staff at Flarion Technologies until August 2001 before joining the ECE Department at the University of Illinois at Urbana- Champaign. Prof. Viswanath is a recipient of the Eliahu Jury Award from the Electrical Engineering and Computer Science Department of the University of California, Berkeley (2000), the Bernard Friedman Award from the Mathematics Department of the University of California, Berkeley (2000), and the NSF CAREER Award (2003). He is serving as Associate Editor for Communications for IEEE TRANSACTIONS ON INFORMATION THEORY for the period 2006-2008. 